\title{
Systematic First Principles
}

\author{
Configuration-Interaction Calculations of \\ Linear Optical Absorption Spectra in Silicon \\ Hydrides : $\mathrm{Si}_{2} \mathrm{H}_{2 n}(n=1-3)$ \\ Pritam Bhattacharyya, ${ }^{*}$ Deepak Kumar Rai, ${ }^{*}$ and Alok Shukla* \\ Department of Physics, Indian Institute of Technology Bombay, Powai, Mumbai 400076, \\ India
}

E-mail: pritambhattacharyya01@gmail.com; dkriitb@gmail.com; shukla@phy.iitb.ac.in

\begin{abstract}
We have performed first principles electron-correlated calculations employing large basis sets to optimize the geometries, and to compute linear optical absorption spectra of various low-lying conformers of silicon hydrides: $\mathrm{Si}_{2} \mathrm{H}_{2 n}, n=1,2,3$. The geometry optimization for various isomers was carried out at the coupled-cluster singles-doubles-perturbative-triples $[\operatorname{CCSD}(\mathrm{T})]$ level of theory, while their excited states and absorption spectra were computed using a large-scale multi-reference singles-doubles configuration-interaction (MRSDCI) approach, which includes electron-correlation effects at a sophisticated level. Our calculated spectra are the first ones for $\mathrm{Si}_{2} \mathrm{H}_{2}$ and $\mathrm{Si}_{2} \mathrm{H}_{4}$ conformers, while for $\mathrm{Si}_{2} \mathrm{H}_{6}$ we obtain excellent agreement with the experimental measurements, suggesting that our computational approach is reliable. Our calculated absorption spectra exhibit a strong structure-property relationship, suggesting the possibility of identifying various conformers based on their optical absorption fingerprints. Furthermore, we have also performed geometry optimization for the selected optically excited states, providing us insights into their character.
\end{abstract}




\section{Introduction}

Silicon is not only one of the most abundant elements on earth, it is also technologically one of the most important ones, with almost the entire semiconductor industry based upon it. Being in the same group of the periodic table as carbon, it shares many chemical characteristics with it. However, unlike carbon, it is not known to participate in $s p^{2}$ hybridization, as a result of which it has no planar graphite-like allotrope. However, there are indications that a quasi-planar allotrope of silicon named silicene can be isolated on substrates. ${ }^{1-4}$ Silicon and its compounds have fascinated physicists and chemists alike, over the years, both from a fundamental as well as from the applied points of view. ${ }^{5-8}$ There has always been a lot of interest in the field of hydrides of various substances, and silicon is no exception. ${ }^{9-13}$ The study of hydrides of silicon is important in many ways: (a) during the formation of silicon thin films using plasma-enhanced chemical vapor deposition, various types of hydrides of silicon are produced, which needs to be understood, ${ }^{14-16}$ (b) degradation of silicon based electronic devices happens normally through hydrogenation of silicon caused by atmospheric moisture, ${ }^{17}$ and (c) hydrogenated amorphous silicon is used extensively in thin film solar cells. ${ }^{17}$ Functionalization of silicene films grown on metal substrates by hydrogenation, with the aim of device applications, is an active area of research these days. ${ }^{18,19}$ Furthermore, the process of hydrogenation in silicon is fascinating from a fundamental point of view, given that the study of hydrogenated carbon in form of hydrocarbons is such a mature field.

Unsaturated silicon hydrides such as $\mathrm{SiH}, \mathrm{SiH}_{2}$, and $\mathrm{SiH}_{3}$ etc. have been the subject of a number of experimental investigations because of their importance in astrophysics, due to their presence in space. ${ }^{20-22}$ Silicon hydrides of the form $\mathrm{Si}_{2} \mathrm{H}_{2 n}, \mathrm{n}=1-3$ have also been extensively studied both experimentally, ${ }^{23-27}$ and by means of high-level theory, ${ }^{25,26,28-41}$ because of their ability to form mono- and dibridged hydrides, as well as multiple bonds of silicon. The carbon analog of $\mathrm{Si}_{2} \mathrm{H}_{2}$ is acetylene, which is a triply-bonded system, while that of $\mathrm{Si}_{2} \mathrm{H}_{4}$ is ethylene, which contains a carbon-carbon double bond. Therefore, the question arises do $\mathrm{Si}_{2} \mathrm{H}_{4}$ and $\mathrm{Si}_{2} \mathrm{H}_{2}$ similarly have silicon-silicon double, and triple bonds, respectively? And, what similarities, if any, does $\mathrm{Si}_{2} \mathrm{H}_{6}$ (disilane) have with $\mathrm{C}_{2} \mathrm{H}_{6}$ (ethane)?

Measurements on $\mathrm{Si}_{2} \mathrm{H}_{2}$ have been performed by several groups. ${ }^{23-25,27,42}$ Bogey et al. ${ }^{24,25,42}$ performed millimeter- and submillimeter-wave spectroscopy measurements on $\mathrm{Si}_{2} \mathrm{H}_{2}$ produced in silaneargon plasma. Ruscic and Berkowitz ${ }^{23}$ produced several $\mathrm{Si}_{2} \mathrm{H}_{n}$ hydrides, including $\mathrm{Si}_{2} \mathrm{H}_{2}$, by reacting fluorine with $\mathrm{Si}_{2} \mathrm{H}_{6}$, and reported photoionization mass spectrometric measurements on it. Recently, 
Mohapatra et al. ${ }^{27}$ synthesized carbene stabilized $\mathrm{Si}_{2} \mathrm{H}_{2}$, and reported measurements of its UV-Vis spectrum. Several first-principles quantum chemical studies of the structure and bonding in $\mathrm{Si}_{2} \mathrm{H}_{2}$ have been performed by several groups. Lischka and Köhler ${ }^{36}$ studied this molecule at the SCF and CEPA-2 level, and were the first ones to predict that in the ground state, the molecule has a dibridged structure $\left(\mathrm{Si}\left(\mathrm{H}_{2}\right) \mathrm{Si}\right)$ with $C_{2 v}$ symmetry. This prediction was also verified in the experiments of Bogey et al. ${ }^{24,25}$ People also believed that the second most stable isomer of $\mathrm{Si}_{2} \mathrm{H}_{2}$ is disilavinylidene $\left(\mathrm{H}_{2} \mathrm{SiSI}\right)$ having $C_{2 v}$ symmetry, while the trans-bent structure (HSiSiH) with $C_{2 h}$ symmetry being the only other minimum. ${ }^{37}$ Later on, the structure of $\mathrm{Si}_{2} \mathrm{H}_{2}$ was re-investigated by Colegrove and Schaefer, ${ }^{33}$ and by Grev and Schaefer ${ }^{37}$ and they found another higher minimum, corresponding to a monobridged structure $(\mathrm{Si}(\mathrm{H}) \mathrm{SiH})$ with $C_{s}$ symmetry, confirmed in the experiment of Cordonnier et al. ${ }^{42}$ Sax and Kalcher, using a first-principles pseudopotential based multi-configuration-self-consistent field (MCSCF) approach, computed the enthalpies of formation of a number of hydrides of silicon, including various isomers of $\mathrm{Si}_{2} \mathrm{H}_{2} \cdot{ }^{29}$ Sannigrahi and Nandi studied the bonding properties of various isomers of $\mathrm{Si}_{2} \mathrm{H}_{2}$ using an ab initio self-consistent-field (SCF) approach. ${ }^{38}$ Jursic performed an extensive first principles study of the potential energy surface of $\mathrm{Si}_{2} \mathrm{H}_{2}$ using density-functional theory (DFT), as well as by employing second-order Møller Plesset perturbation theory combined with Gaussian-1/Gaussian-2/Gaussian-3 approaches. More recently, Schaefer and coworkers ${ }^{28}$ computed the electron affinities several hydrides of silicon, including $\mathrm{Si}_{2} \mathrm{H}_{2}$.

The subject of doubly bonded silicon compounds silenes was reviewed by Raabe and Michl. ${ }^{43}$ In the year 1981, West, Fink and Michl ${ }^{44}$ experimentally stabilized the silicon-silicon doubly bonded compound tetramesityldisilene $\mathrm{Si}_{2} \mathrm{R}_{4}$, by using the bulky 2,4,6- trimethylphenyl (mesityl) group, denoted here as R. These authors, also measured its UV-Vis absorption spectrum. ${ }^{44}$ However, the first synthesis of doublybonded molecule disilene $\left(\mathrm{Si}_{2} \mathrm{H}_{4}\right)$, was reported by Ruscic and Berkowitz ${ }^{23}$ in 1991, who also reported the measurements of its ionization potential. Using infrared spectroscopy, supported by first principles DFT calculations, Andrews and Wang concluded that $\mathrm{Si}_{2} \mathrm{H}_{4}$ has a $C_{2 h}$ structure. ${ }^{22}$ Sari et al. ${ }^{26}$, based upon rotational spectrum measurements of $\mathrm{Si}_{2} \mathrm{H}_{4}$ by means of Fourier Transform Microwave (FTM) spectroscopy, supported by sophisticated coupled-cluster calculations, concluded that the molecule has a monobridged structure. Later on, the same group presented another experimental and theoretical study on $\mathrm{Si}_{2} \mathrm{H}_{4}$ molecule, and concluded that indeed the monobridged isomer was most abundant. However, several unidentified spectral lines in the experiment could imply the presence of a dibridged isomer as 
well. ${ }^{41}$ As far as theoreticians are concerned, there appears to be a general agreement that the trans-bent structure of disilene corresponds to the true ground state, while the monobridged structure is believed to be the next higher energy isomer. ${ }^{22,26,28,30,34,35,40,41,45-48}$ Silysilylene isomer, with the structure $\mathrm{H}_{3} \mathrm{SiSiH}$, has been computed to be the third higher energy structure. ${ }^{26,30,34,35,41,47,48}$

Disilane $\left(\mathrm{Si}_{2} \mathrm{H}_{6}\right)$, which is the structural analog of ethane $\left(\mathrm{C}_{2} \mathrm{H}_{6}\right)$, is a stable compound existing in gas phase at room temperature, and has been known for a long time. As a result, a number of experimental and theoretical studies have been performed on it. The infrared spectrum of gas phase disilane was observed by Gutowsky and Stejskal from 350 to $4000 \mathrm{~cm}^{-149}$, as well as by Andrews and Wang by reacting laser-ablated silicon atoms with molecular hydrogen to form the silicon hydrides ${ }^{22}$. Itoh et al. experimentally studied the vacuum ultraviolet absorption cross sections of disilane. ${ }^{50}$ Several authors experimentally studied the photo-electron spectra of silanes $\left(\mathrm{Si}_{n} \mathrm{H}_{2 \mathrm{n}+2}\right)$, and measured their ionization potentials. ${ }^{51,52}$ As far as theoretical calculations on disilane are concerned, in 1976, geometry optimization was performed by Blustin ${ }^{53}$, and Pople and coworkers ${ }^{54}$. In the same year, the valence electronic structure and internal rotation barrier of the molecule was computed by Nicolas, Barthelat, and Durand using a pseudo-potential method. ${ }^{55}$ In 1981, Ratner and coworkers performed electronic structure calculations employing a Hartree-Fock-Slater (HFS) procedure, based upon the local-density functional approach. ${ }^{56}$ Photolytic fragmentation was studied by Janoschek and coworkers using a pseudopotential method. ${ }^{57}$ In 1986, the geometries and the single point energies of many singly bonded silicon compounds, including disilane, were computed by Schleyer and coworkers using HF and MP4 level of theory. ${ }^{58}$ Sax performed local pseudopotential calculations to optimize the ground state geometry of disilane. ${ }^{30}$ Using ab initio propagator theory, ionization potentials of various silicon hydrides, including $\mathrm{Si}_{2} \mathrm{H}_{6}$, were theoretically computed by Ortiz and Mintmire. ${ }^{59}$ Using a first-principles configuration interaction (CI) approach, Kawai et al. ${ }^{60}$ computed the ultraviolet photoabsorption spectrum of disilane. Rohlfing and Louie computed the optical absorption spectrum of disilane by solving the Bethe-Salpeter equation, within a first-principles formalism based upon density-functional theory (DFT), with quasiparticle effects included using the GW approximation. ${ }^{61}$

In this work we undertake a comprehensive study of structural stability and optical properties of three hydrides of silicon dimer, namely, $\mathrm{Si}_{2} \mathrm{H}_{2}, \mathrm{Si}_{2} \mathrm{H}_{4}$ and $\mathrm{Si}_{2} \mathrm{H}_{6}$ using state-of-the-art correlated-electron first principles electronic structure methodology. Geometry optimization for all the molecules considered was carried out using the coupled-cluster singles-doubles-triples (CCSD $(\mathrm{T})$ ) level of theory, using 
large basis sets including polarization functions. The optical absorption spectra of various clusters were computed using the multi-reference singles-doubles configuration interaction (MRSDCI) approach, which has been used in our group to study the optical properties of a variety of systems such as atomic clusters, ${ }^{62-65}$ conjugated polymers, ${ }^{66-71}$ and graphene quantum dots. ${ }^{72,73}$ We would like to emphasize that the first-principles electronic structure studies of Si based systems are more computationally expensive as compared to similar studies of clusters made up of smaller atoms, simply because Si has more electrons, thereby requiring larger basis sets, and hence more computer memory and time. For $\mathrm{Si}_{2} \mathrm{H}_{2}$ and $\mathrm{Si}_{2} \mathrm{H}_{4}$, the linear optical absorption spectra were computed for a number of isomers, with the aim of understanding the influence of geometry on the optical properties of these molecules. The relation between absorption spectra and geometry can be used for the detection and identification of these molecules in optical experiments. To achieve better understanding of the nature of optically excited states, we have also performed geometry optimization on a few selected excited states. Our calculations reveal that higher energy excited states of various clusters have significantly different relaxed geometries as compared to the ground state.

So far, optical absorption experiments on $\mathrm{Si}_{2} \mathrm{H}_{2}$ and $\mathrm{Si}_{2} \mathrm{H}_{4}$ have not been performed. Therefore, our theoretical calculations of their absorption spectra will be useful in guiding future experimental efforts on these systems. For the case of disilane, our calculated optical absorption spectrum is in very good agreement with the experimental measurements of Itoh et al. ${ }^{50}$ as well as with the BetheSalpeter equation based theoretical calculations of Rohlfing and Louie ${ }^{61}$. This excellent agreement of our calculations with the experiments for disilane gives us confidence that our calculations on $\mathrm{Si}_{2} \mathrm{H}_{2}$ and $\mathrm{Si}_{2} \mathrm{H}_{4}$ should be equally accurate.

\section{Theory And Computational Details}

\section{General Methodology}

All the calculations were carried out using a wave-function-based first-principles methodology, employing the molecular Born-Oppenheimer Hamiltonian, in which the orbitals of molecules are expressed as linear combinations of Cartesian-Gaussian-type basis functions. Such a quantum-chemical electronic structure approach has been implemented in a number of program packages, and in this work we used the packages 
PSI4 ${ }^{74}$ and MELD. ${ }^{75}$ The geometries of the silicon hydride $\left(\mathrm{Si}_{2} \mathrm{H}_{2 \mathrm{n}}, \mathrm{n}=1-3\right)$ molecules studied in this work were optimized using the coupled-cluster singles-doubles-perturbative-triples (CCSD(T)) approach, as implemented in the program package PSI4, ${ }^{74}$ utilizing the correlation-consistent polarized valencetriple-zeta (cc-pVTZ) basis sets. Once the ground state geometries were determined for each isomer, calculations of their optical absorption spectra were performed. For the purpose, calculations of the excited states of the molecules was performed using the multireference singles-doubles configurationinteraction (MRSDCI) approach, as implemented in the MELD package. ${ }^{75}$ To perform the MRSDCI calculations, first we transform the Hamiltonian from the atomic orbital (AO) representation consisting of Cartesian Gaussian basis functions, to the molecular orbital (MO) representation. This is achieved by obtaining the MOs of the concerned isomer by performing restricted Hartree-Fock (RHF) calculations on its optimized geometry, and then transforming the one- and two-electron integrals from the AO to the MO representation. Next, using the transformed Hamiltonian, a singles-doubles CI (SDCI) calculation is performed using an appropriate single reference wave function, both for the ground state, and the excited states. The many-body wave functions obtained from the SDCI calculation are used for computing its optical absorption spectrum. Next, the wave functions contributing to various peaks are analyzed, and the configuration state functions (CSFs) making significant contributions to the corresponding excited states are included as references for the next set of MRSDCI calculation. This procedure is iterated until the calculated optical absorption spectrum of the isomer converges. At every stage of the calculation, point-group and spin symmetries are fully utilized, thus our ground and excited state wave functions are also eigenfunctions of the corresponding point-group projection operator, and total-spin operator. For details of the MRSDCI approach adopted in this work, we refer the reader to our earlier works. ${ }^{62-73}$

The optical absorption spectrum $\sigma(\omega)$, is calculated within the electric-dipole approximation, using the formula

$$
\sigma(\omega)=4 \pi \alpha \sum_{i} \frac{\omega_{i 0}|\langle i|\hat{\boldsymbol{e}} \cdot \boldsymbol{r}| 0\rangle|^{2} \gamma^{2}}{\left(\omega_{i 0}-\omega\right)^{2}+\gamma^{2}}
$$

where $\omega, \hat{\boldsymbol{e}}, \boldsymbol{r}$, and $\alpha$, respectively denote the frequency of the incident light, polarization direction of the incident light, the position operator, and the fine structure constant. Furthermore, $\omega_{i 0}$ is the energy difference (in frequency units) between the ground state (0) and the $i^{\text {th }}$ excited state, while $\gamma$ is the uniform lined width associated with each excited state energy level. The summation over $i$ in Eq. 1 
includes an infinite number of excited states, however, in our calculations we restricted the sum to those excited states which are no more than $10 \mathrm{eV}$ higher than the ground state.

The absorption spectrum calculated using the formalism described above corresponds to vertical excitations, i.e., those optical excitations during which the geometry of the system has no time to relax. However, eventually the system undergoes relaxation, and the molecular geometry of the excited state changes as compared to that of the ground state. In order to understand this aspect of the excited states of various isomers, for a few important states, we performed geometry optimization. For the purpose, CI-singles (CIS)-based excited-state geometry-optimization approach, as implemented in Gaussian-16 program, ${ }^{76}$ was employed. For visualizing the molecular orbitals we used GaussView $6{ }^{77}$, and for plotting the molecular geometries, XCrySDen software ${ }^{78}$ was employed.

\section{Computational parameters}

In this section we discuss our choices of three computational parameters, namely: (a) Gaussian basis functions, (b) orbital basis set, and (c) many-particle configurations.

\section{Choice of the Gaussian basis set}

A wide variety of Gaussian basis functions are available, depending upon the task at hand. For example, for ground state geometry many workers prefer Pople basis sets of the type 6-311, which are moderately sized, and yield good results for ground state properties. However, we wanted to use a basis set which can be utilized both for ground state geometry optimization, as well as excited state calculations needed for computation of the optical absorption spectra. Therefore, in these calculations we adopted correlation consistent Dunning basis set cc-pVTZ, which is known to yield good results both for the ground and excited state calculations. However, in order to ensure the convergence of our computed optical spectrum, we also computed it by using larger aug-cc-pVTZ basis set containing several diffuse exponents for an isomer (see Fig. S9 of the Supporting Information), and found that except for changes in absolute intensities of the peaks, no significant changes in peak locations were observed. Thus, cc-pVTZ basis set

was used both for Si and $\mathrm{H}$ atoms, during geometry optimization performed at the CCSD $(\mathrm{T})$ level, ${ }^{74}$ and also for excited state calculations performed using the MRSDCI approach. In an earlier work from our group dealing with the optical absorption of bare Al clusters, we had also used the cc-pVTZ basis 
set for $\mathrm{Al}$ atoms. ${ }^{65}$ Given the quality of this basis set, we expect our calculations to be fairly accurate.

\section{Molecular Orbital basis}

It is a well-known fact that the computational effort at the CI level scales as $\approx N^{6}$, where $N$ is the total number of molecular orbitals employed in the CI calculations. Thus, the computation time increases steeply, with the increasing size of the MO set used in the CI calculations. Therefore, to keep the calculations tractable, we employed the frozen-core approximation, thereby not only reducing the orbital basis size, but also the number of electrons employed in the CI calculations to just four per atom, corresponding to the valence electrons of each Si atom. In our earlier works on various clusters, we carefully studied the influence of frozen core approximation on the computed optical absorption spectra, and found that the results were unaffected by it. ${ }^{62-65}$ As far as virtual orbitals are concerned, we did not discard any orbital in MRSDCI calculations for all the molecules except for mono-briged (Si-H$\mathrm{SiH})$, disilavinylidene $\left(\mathrm{Si}-\mathrm{SiH}_{2}\right)$, and disilane $\left(\mathrm{H}_{3} \mathrm{Si}_{-} \mathrm{SiH}_{3}\right)$, for which we retained all those virtual orbitals whose energies were less than one Hartree. This "one Hartree" cutoff is computationally sound because we are interested in optical excitations whose energy is much smaller. Nevertheless, we would like to emphasize that during geometry optimization performed using the $\mathrm{CCSD}(\mathrm{T})$ method, ${ }^{74}$ each calculation was carried out at the all-electron level, without truncating the available MO set.

\section{Size of the CI expansion.}

As discussed earlier, we initiate the MRSDCI calculations with a small number of reference configurations, and compute the optical absorption spectrum of the system concerned. By analyzing the excited states contributing to the peaks in the computed spectrum, we increase the number of reference configurations, and perform next level of MRSDCI calculation leading to a new optical absorption spectrum. This procedure is iterated until the calculated absorption spectrum exhibits reasonable convergence. Whether to include a given configuration in the list of reference configurations is based upon the magnitude of its coefficient in the many-particle wave function of an excited state contributing to a significant peak in the calculated absorption spectrum. In Fig. 1 we demonstrate this procedure for the case of monobridged isomer of $\mathrm{Si}_{2} \mathrm{H}_{4}$ molecule. Denoting the total number of reference configurations as $N_{\text {ref }}$, and the total number of CSFs in that CI expansion as $N_{\text {total }}$, the three MRSDCI calculations presented in Fig. 1 were performed using $N_{\text {ref }}=13\left(N_{\text {total }}=851933\right), N_{\text {ref }}=32\left(N_{\text {total }}=1975358\right)$, 
and $N_{\text {ref }}=41\left(N_{\text {total }}=2506254\right)$, respectively. From the plotted spectra it is obvious that MRSDCI_3 calculation has converged to an acceptable level, both qualitatively and quantitatively, when compared to the MRSDCI_2 calculation.

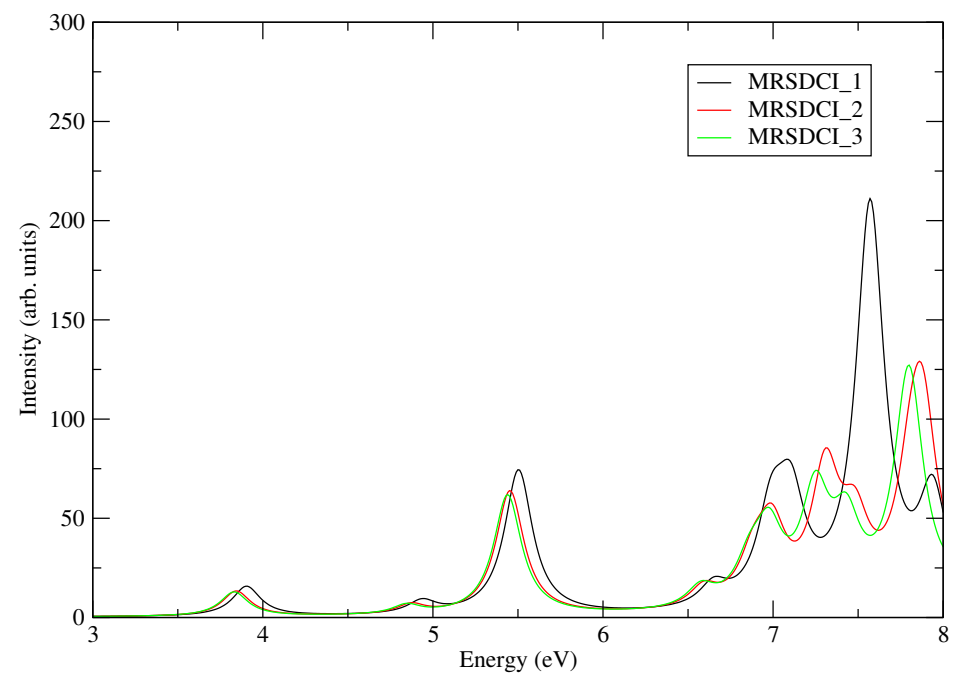

Figure 1: Convergence of the MRSDCI optical absorption spectrum of the monobridged isomer of $\mathrm{Si}_{2} \mathrm{H}_{4}$ molecule, with respect to the increasing number of reference configurations $\left(N_{\text {ref }}\right)$. Calculations labeled MRSDCI_1, MRSDCI_2, and MRSDCI_3 were performed using 13, 32, and 41 reference configurations, respectively.

\section{Results and Discussion}

In Table 1 we present our results on the energetics corresponding to the optimized geometries of various molecules, to be discussed in detail later on. An inspection of Table 1 reveals that all the isomers studied in this work have large binding energies in the range $10.62-24.23 \mathrm{eV}$, implying that they will be bound at the ambient conditions. Furthermore, for each isomer we also performed vibrational frequency analysis, and no imaginary frequencies were obtained. This suggests that all the conformers studied in this work represent stable structures.

In Table 2 we present the sizes of the CI matrices involved in the MRSDCI calculations performed on these molecules, for various irreducible representations (irreps) corresponding to their ground, and excited state, wave functions. From the sizes of the CI matrices, it is obvious that our calculations were large scale, and, therefore, account for electron correlation effects in an adequate manner. Next, we discuss the calculated ground state geometries, and the optical absorption spectra, of various isomers of silicon hydride molecules. We present the results of our calculations on $\mathrm{Si}_{2} \mathrm{H}_{6}$ molecule first, because 
it is the only molecule studied in this work for which both the experimental, as well theoretical, results are available. Therefore, comparing our results with those will allow us to benchmark our approach, and make it trustworthy for other molecules for which no earlier data on optical absorption is available.

Table 1: Total ground state (GS) energies (in Hartree), point group, symmetry of the ground state, relative energies, correlation energies, and the binding energies of the hydrogenated silicon conformers computed using the $\operatorname{CCSD}(\mathrm{T})$ approach, using the cc-pVTZ basis sets. The correlation energy indicates the difference of energies computed using the $\operatorname{CCSD}(\mathrm{T})$ and HF levels of theory. To obtain the total binding energy of a system presented in the last column, we have also taken care of the basis set superposition error (BSSE) using the counterpoise correction.

\begin{tabular}{cccccccc}
\hline Molecule & Conformer & $\begin{array}{c}\text { Point } \\
\text { group }\end{array}$ & $\begin{array}{c}\text { Symmetry } \\
\text { of the GS } \\
\text { wave function }\end{array}$ & $\begin{array}{c}\text { Total energy } \\
(\mathrm{Ha})\end{array}$ & $\begin{array}{c}\text { Relative } \\
\text { energy } \\
(\mathrm{eV})\end{array}$ & $\begin{array}{c}\text { Correlation } \\
\text { energy }(\mathrm{eV})\end{array}$ & $\begin{array}{c}\text { Binding } \\
\text { energy } \\
(\mathrm{eV})\end{array}$ \\
\hline $\mathrm{Si}_{2} \mathrm{H}_{2}$ & Dibridged disilyne & $\mathrm{C}_{2 \mathrm{v}}$ & ${ }^{1} \mathrm{~A}_{1}$ & -579.33918 & 0.0 & 10.58 & 11.38 \\
$\mathrm{Si}_{2} \mathrm{H}_{2}$ & Monobridged & $\mathrm{C}_{\mathrm{s}}$ & ${ }^{1} \mathrm{~A}^{\prime}$ & -579.32320 & 0.4348 & 10.74 & 10.93 \\
$\mathrm{Si}_{2} \mathrm{H}_{2}$ & Disilavinylidene & $\mathrm{C}_{2 \mathrm{v}}$ & ${ }^{1} \mathrm{~A}_{1}$ & -579.31788 & 0.5796 & 10.24 & 10.86 \\
$\mathrm{Si}_{2} \mathrm{H}_{2}$ & Trans-bent & $\mathrm{C}_{2 \mathrm{~h}}$ & ${ }^{1} \mathrm{~A}_{\mathrm{g}}$ & -579.30975 & 0.8008 & 10.94 & 10.62 \\
$\mathrm{Si}_{2} \mathrm{H}_{4}$ & Disilene & $\mathrm{C}_{2 \mathrm{~h}}$ & ${ }^{1} \mathrm{~A}_{\mathrm{g}}$ & -580.55905 & 0.0 & 11.41 & 17.32 \\
$\mathrm{Si}_{2} \mathrm{H}_{4}$ & Monobridged & $\mathrm{C}_{1}$ & ${ }^{1} \mathrm{~A}$ & -580.54854 & 0.2859 & 11.41 & 17.01 \\
$\mathrm{Si}_{2} \mathrm{H}_{4}$ & Silylsilylene & $\mathrm{C}_{\mathrm{s}}$ & ${ }^{1} \mathrm{~A}^{\prime}$ & -580.54823 & 0.2944 & 11.06 & 17.05 \\
$\mathrm{Si}_{2} \mathrm{H}_{6}$ & & & & & & & \\
\hline
\end{tabular}


Table 2: Point group symmetry employed in the calculations, along with the total number of configurations $\left(N_{\text {total }}\right)$ in the MRSDCI expansion, aimed at computing the optical absorption spectra of various hydrogenated silicon conformers. In all the calculations, cc-pVTZ basis sets were used both for $\mathrm{Si}$ and $\mathrm{H}$ atoms.

\begin{tabular}{|c|c|c|c|c|}
\hline Molecule & Conformer & Point group used & Symmetry & $N_{\text {total }}$ \\
\hline \multirow[t]{3}{*}{$\mathrm{Si}_{2} \mathrm{H}_{2}$} & Dibridged disilyne & $\mathrm{C}_{2 \mathrm{v}}$ & ${ }^{1} \mathrm{~A}_{1}$ & 1458235 \\
\hline & & & ${ }^{1} \mathrm{~B}_{1}$ & 1951202 \\
\hline & & & ${ }^{1} \mathrm{~B}_{2}$ & 1105180 \\
\hline $\mathrm{Si}_{2} \mathrm{H}_{2}$ & Monobridged & $\mathrm{C}_{1}$ & ${ }^{1} \mathrm{~A}$ & 1681403 \\
\hline $\mathrm{Si}_{2} \mathrm{H}_{2}$ & Disilavinylidene & $\mathrm{C}_{1}$ & ${ }^{1} \mathrm{~A}$ & 2526917 \\
\hline \multirow[t]{3}{*}{$\mathrm{Si}_{2} \mathrm{H}_{2}$} & Trans-bent & $\mathrm{C}_{2 \mathrm{~h}}$ & ${ }^{1} \mathrm{~A}_{\mathrm{g}}$ & 221709 \\
\hline & & & ${ }^{1} \mathrm{~A}_{\mathrm{u}}^{0}$ & 2818861 \\
\hline & & & ${ }^{1} \mathrm{~B}_{\mathrm{u}}$ & 2643120 \\
\hline \multirow[t]{3}{*}{$\mathrm{Si}_{2} \mathrm{H}_{4}$} & disilene & $\mathrm{C}_{2 \mathrm{~h}}$ & ${ }^{1} \mathrm{~A}_{\mathrm{g}}$ & 42206 \\
\hline & & & ${ }^{1} \mathrm{~A}_{\mathrm{u}}^{\mathrm{o}}$ & 359342 \\
\hline & & & ${ }^{1} \mathrm{~B}_{\mathrm{u}}$ & 501900 \\
\hline $\mathrm{Si}_{2} \mathrm{H}_{4}$ & monobridged & $\mathrm{C}_{1}$ & ${ }^{1} \mathrm{~A}$ & 2506254 \\
\hline $\mathrm{Si}_{2} \mathrm{H}_{4}$ & silylsilylene & $\mathrm{C}_{1}$ & ${ }^{1} \mathrm{~A}$ & 3404169 \\
\hline \multirow[t]{3}{*}{$\mathrm{Si}_{2} \mathrm{H}_{6}$} & Disilane & $\mathrm{C}_{2 \mathrm{~h}}$ & ${ }^{1} \mathrm{~A}_{\mathrm{g}}$ & 20621 \\
\hline & & & ${ }^{1} \mathrm{~A}_{\mathrm{u}}$ & 1634632 \\
\hline & & & ${ }^{1} \mathrm{~B}_{\mathrm{u}}$ & 2038895 \\
\hline
\end{tabular}

\section{Disilane $\mathrm{Si}_{2} \mathrm{H}_{6}$}

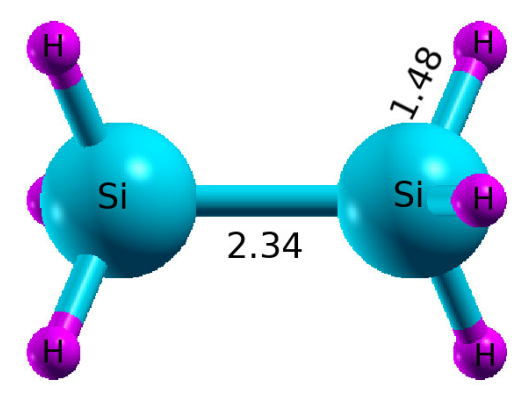

Figure 2: Ground state geometry of disilane $\left(\mathrm{H}_{3} \mathrm{Si}_{-} \mathrm{SiH}_{3}\right)$, optimized using the $\mathrm{CCSD}(\mathrm{T})$ method, and the cc-pVTZ basis set. All the bond lengths are in $\AA$ unit.

As far as disilane $\left(\mathrm{Si}_{2} \mathrm{H}_{6}\right)$ is concerned, it has only one stable conformer with a three-dimensional $\mathrm{sp}^{3}$-hybridized structure, and the configuration $\mathrm{H}_{3} \mathrm{Si}_{-} \mathrm{SiH}_{3}$, similar to the case of ethane $\left(\mathrm{C}_{2} \mathrm{H}_{6}\right)$. In 
this structure, again similar to the case of ethane, the hydrogen atoms are arranged in a staggered configuration, instead of an eclipsed one, leading to the ground state point-group symmetry $D_{3 d}$. Our optimized geometry obtained using the $\operatorname{CCSD}(\mathrm{T})$ method, and cc-pVTZ basis set, depicted in Fig. 2, has only two unique bond lengths: (a) Si-Si bond length of $2.34 \AA$, and (b) Si-H bond length 1.48 $\AA$, along with the H-Si-H and H-Si-Si bond angles of $108.6^{\circ}$ and $110.3^{\circ}$. Our calculated geometry parameters are in good agreement with the experimental, ${ }^{79,80}$ as well as theoretical values computed by other authors. ${ }^{30,55,59}$ The atomic coordinates corresponding to our optimized ground state geometries are presented in Table S16 of the Supporting Information.

Our photoabsorption spectrum of disilane is presented in Fig. 3, while the optimized geometries of a couple of excited states are given in Fig. S17 of the Supporting Information. It is obvious from the absorption spectrum that it consists of six well-separated peaks of fairly strong intensities, except for the last one, which is comparatively weaker.

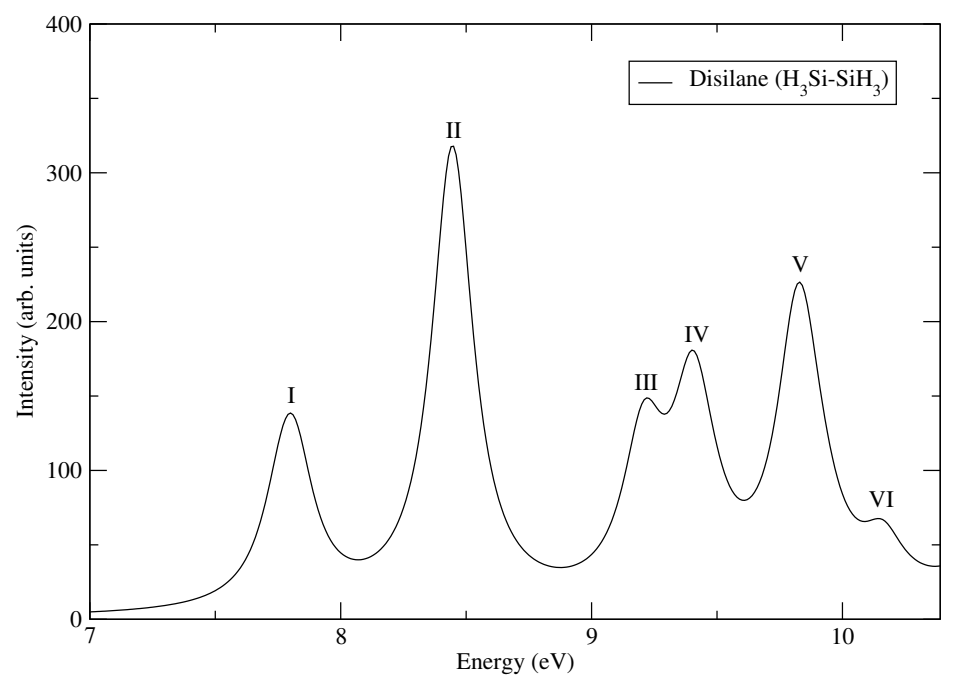

Figure 3: Optical absorption spectrum of disilane computed using the MRSDCI method, and the cc-pVTZ basis set. For plotting the spectrum, we assumed a uniform line-width of $0.1 \mathrm{eV}$.

The optical absorption in disilane starts at fairly high energies, as compared to previously discussed molecules, with a moderately intense peak near $7.80 \mathrm{eV}$. This peak is due to two close-lying excited states, both whose wave function are dominated by the singly-excited configuration $|H \rightarrow(L+2)\rangle$, where the orbital $L+2$ is doubly degenerate. The relaxed geometry of this state is twisted with respect to the ground state about the Si-Si bond axis. Furthermore, Si-Si bond is a bit longer $(2.60 \AA)$ compared to the ground state, whereas not all the $\mathrm{Si}-\mathrm{H}$ bond lengths are exactly equal, but still close enough to their ground state values. The first absorption is followed by the most intense peak (peak II) of the 
spectrum located at $8.45 \mathrm{eV}$, due to a state whose wave function mainly consists of the single excitation $|H \rightarrow L\rangle$. In the relaxed structure of this excited state, Si-Si bond is substantially stretched $(3.47 \AA)$ compared to the ground state, but no changes are observed in any of the Si-H bond lengths. The next peak in the spectrum (peak III) occurs at $9.21 \mathrm{eV}$, and is due to a state whose wave function is dominated by almost equal contributions from the two degenerate excitations $|H-1 \rightarrow L+2\rangle$, where orbital $H-1$ is also doubly degenerate, just like orbital $L+2$. Next we have two peaks in the spectrum located at 9.41 $\mathrm{eV}$ and $9.84 \mathrm{eV}$, both of which are due to two closely-lying states each. The first of these peaks (peak IV) derives its main intensity from a state located at $9.40 \mathrm{eV}$, with a small contribution from a state at $9.47 \mathrm{eV}$. The main contribution to oscillator strength of peak $\mathrm{V}$ comes from a state located at $9.82 \mathrm{eV}$, with a much smaller intensity derived from a state located $9.92 \mathrm{eV}$. The wave functions of all the four excited states contributing to peaks IV and V exhibit strong configuration mixing, and are composed of various degenerate combinations of singly-excited configurations $|H-1 \rightarrow L+2\rangle,|H-1 \rightarrow L\rangle$, and $|H-2 \rightarrow L\rangle$ (see Table S8 of Supporting Information). Final peak of the computed spectrum (peak VI) located at $10.16 \mathrm{eV}$ is a relatively weaker one, and is due to a state whose wave function consists mainly of the configuration $|H \rightarrow L+4\rangle$, with a smaller contribution from the excitation $|H-1 \rightarrow L+2\rangle$.

In Table 3 we compare our calculated peak locations to the experimentally measured values of Itoh et al. ${ }^{50}$, and the Bethe-Salpeter equation based calculations of Rohlfing et al., ${ }^{61}$ and find that our results are in very good agreement with the experiments. This level of agreement between our calculations and the experiments for the case of disilane, the largest studied molecule in this work, suggests that our computational methodology is sound and trustworthy.

Table 3: Comparison of the peak locations (in $\mathrm{eV}$ ) in the calculated optical absorption spectra of disilane, with the experimental values reported by Itoh et al., ${ }^{50}$ and the theoretical values reported by Louie et al. ${ }^{61}$ obtained from their Bethe-Salpeter equation based calculations.

\begin{tabular}{ccc}
\hline This work & Expt. $\left(\right.$ Ref. $\left.^{50}\right)$ & Theory $\left(\right.$ Ref. $\left.^{61}\right)$ \\
\hline \hline 7.80 & 7.6 & 7.6 \\
8.45 & 8.4 & 9.0 \\
9.41 & 9.5 & 9.6 \\
9.84 & 9.9 & 9.8 \\
\hline
\end{tabular}




\section{$\mathrm{Si}_{2} \mathrm{H}_{2}$}

$\mathrm{Si}_{2} \mathrm{H}_{2}$ is the smallest member of the $\mathrm{Si}_{2} \mathrm{H}_{2 n}$ class of molecules, several of whose conformers have been studied in the past. ${ }^{33,36-38}$ The five most studied conformers of $\mathrm{Si}_{2} \mathrm{H}_{2}$ molecule are dibridged disilyne, mono-bridged structure, disilavinylidene, trans-bent structure, and the planar dibridged disilyne. The stability analysis of these conformers revealed that all of them are stable, except for the planar dibridged disilyne structure, which corresponds to a saddle point, or a transition state, on the potential energy surface. ${ }^{33,36-38}$ As a result, we restricted the present study to the four stable structures, and we optimized their geometries using the the cc-pVTZ basis set, and the coupled-cluster singles-doubles-perturbativetriples $[\mathrm{CCSD}(\mathrm{T})]$ method, as implemented in the PSI4 computer program. ${ }^{74}$ The optimized geometries are presented in Fig. 4, while Table 1 contains their total and relative energies. From the table it is obvious that the dibridged disilyne is the lowest energy isomer, followed by the monobridged structure which is $0.44 \mathrm{eV}$ higher. Next are two conformers disilavinylidene and trans-bent structure, which are higher in energy by $0.58 \mathrm{eV}$, and $0.80 \mathrm{eV}$, respectively, as compared to the lowest-energy dibridged disilyne conformer. It is obvious that all the higher energy conformers are within $1 \mathrm{eV}$ of the lowestenergy structure, and even closer to each other. Therefore, it will be interesting to see if their optical absorption spectra, which were computed using the MRSDCI approach using these geometries, are different enough to facilitate their optical detection. In the following sections we discuss the optimized geometries and optical absorption spectra of individual conformers.

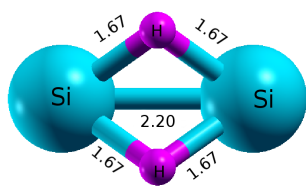

(a) Dibridged disilyne

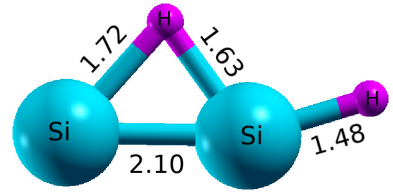

(b) Mono-bridged

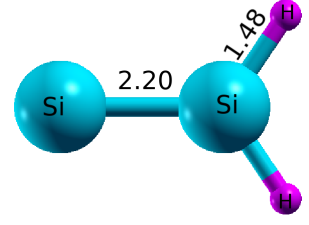

(c) Disilavinylidene

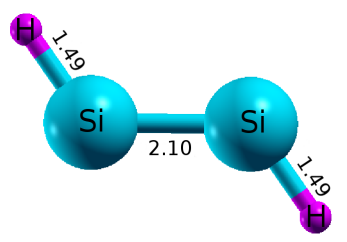

(d) Trans-bent

Figure 4: Ground state geometries of various isomers of $\mathrm{Si}_{2} \mathrm{H}_{2}$ molecule, optimized using the CCSD(T) method, and the cc-pVTZ basis set. All the bond lengths are in $\AA$ unit.

\section{Dibridged disilyne $\left(\mathrm{Si}-\mathrm{H}_{2}-\mathrm{Si}\right)$}

Our optimized structure of dibridged disilyne conformer, as shown in Fig. 4(a), consists of two threecenter Si-H-Si bonds with identical Si-H bond lengths of $1.67 \AA$, which are non-planar leading to the $C_{2 v}$ point-group symmetry, instead of $D_{2 h}$, had they been planar. Furthermore, we obtained the optimized 
Si-Si distance to be $2.20 \AA$, along with Si-H-Si, H-Si-H and H-Si-Si bond angles as $82.5^{\circ}, 72.3^{\circ}$ and $48.8^{\circ}$, respectively, whereas the $\mathrm{HSi}-\mathrm{SiH}$ dihedral angle is $103.4^{\circ}$. Our optimized geometry parameters are in good agreement with the experimental results of Bogey et al. ${ }^{25}$ obtained using millimeter- and submillimeter-wave spectroscopy, as also with their ab initio theoretical results. Our results are also in good agreement with the theoretical calculations reported by Gerv and Schaefer ${ }^{37}$, Adamczyk and Broadbelt ${ }^{81}$, Jursic, ${ }^{39}$ and Sannigrahi and Nandi. ${ }^{38}$ Atomic coordinates corresponding to the optimized ground state geometry are presented in Table S9 of the Supporting Information.

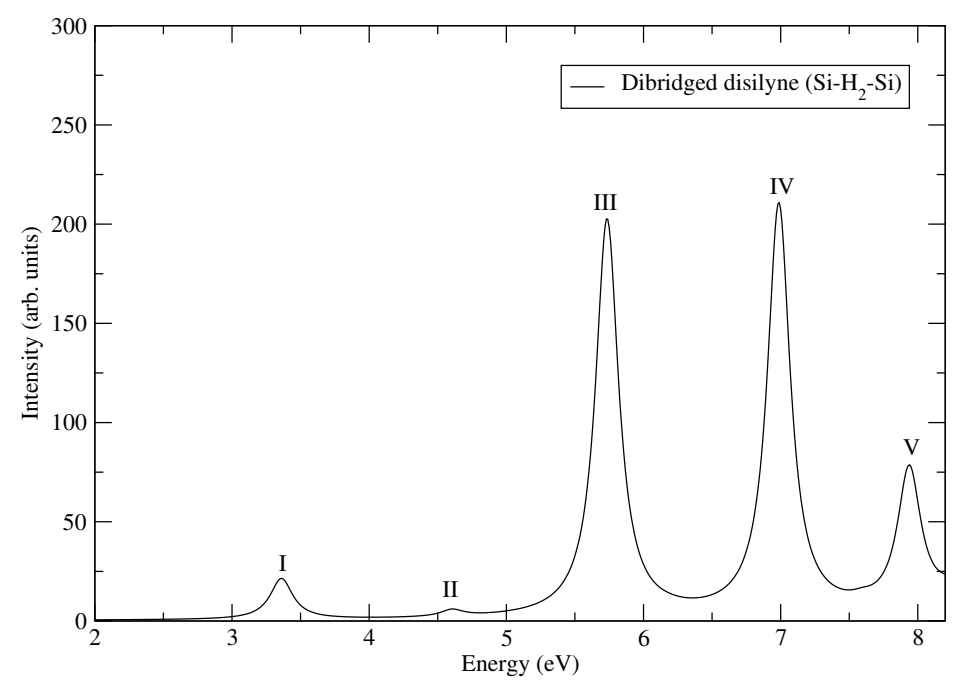

Figure 5: Optical absorption spectrum of non-planar disilyne ( $\left.\mathrm{Si}_{-} \mathrm{H}_{2}-\mathrm{Si}\right)$ conformer, computed using the MRSDCI method, and the cc-pVTZ basis set. For plotting the spectrum, $0.1 \mathrm{eV}$ uniform line-width was used.

The calculated photoabsorption spectrum of this conformer is presented in Fig. 5, while the relaxed excited-state structures corresponding to some of the frontier peaks of the spectrum are given in Fig. S10 of the Supporting Information. The absorption starts with a relatively weak peak near $3.36 \mathrm{eV}$, followed by an even weaker peak at $4.60 \mathrm{eV}$. The first of these peaks (peak I) is due to an excited state whose wave function is dominated by the singly-excited configuration $|H \rightarrow L\rangle$, while that corresponding to the second peak (peak II) consists mainly of the configuration $|H \rightarrow L+2\rangle$. The optimized geometry of the excited state corresponding to peak I is almost similar to the ground-state geometry, except for the elongated Si-Si, and Si-H bond lengths, $2.36 \AA$, and $1.73 \AA$, respectively. These weak peaks are followed by the two of the most intense peaks (peaks III and IV) of the spectrum, located near $5.74 \mathrm{eV}$, and $6.99 \mathrm{eV}$. Peak III is due to two excited states, whose wave functions exhibit significant configuration mixing. The first state at $5.73 \mathrm{eV}$ contributes dominantly to the intensity of the peak, and its wave function consists mainly of configurations $|H-1 \rightarrow L\rangle$ and $|H \rightarrow L+4\rangle$. The wave function of the 
second state around $5.77 \mathrm{eV}$ is dominated by doubly-excited configurations $|H \rightarrow L+3 ; H \rightarrow L+3\rangle$ and $|H \rightarrow L ; H \rightarrow L\rangle$. The optimized geometry of the excited state located at 5.73 eV looks like a monobridged structure, with the $\mathrm{Si}-\mathrm{H}$ bond distances $1.72 \AA$, and $1.68 \AA$, whereas the other H-atom is connected with a Si-atom with a bond length $1.51 \AA$. Furthermore, the Si-Si bond length is elongated compared to the ground-state, with a bond distance $2.45 \AA$.

The wave function of the excited state contributing to peak IV is dominated by single excitations $|H \rightarrow L+4\rangle$ and $|H-3 \rightarrow L+1\rangle$. The optimized geometry of this peak has a trans-like structure with Si-Si and Si-H bond lengths, $2.30 \AA$, and $1.50 \AA$, respectively. The final peak of the spectrum (peak V) located close to $7.93 \mathrm{eV}$, is weaker compared to these two, and is due to two excited states located at $7.91 \mathrm{eV}$ and $7.94 \mathrm{eV}$. The wave function of the first of these is dominated by the double excitation $|H-1 \rightarrow L+3 ; H-1 \rightarrow L+3\rangle$, while that of the second one derives main contributions from single excitations $|H-1 \rightarrow L+2\rangle$ and $|H-2 \rightarrow L+1\rangle$. Detailed information about all the excited states contributing to the peaks in the spectra is presented in Table S1 of the Supporting Information, while its Fig. S1 contains the plots of frontier MOs participating in the optical absorption.

\section{Monobridged (Si-H-SiH)}

The monobridged conformer of $\mathrm{Si}_{2} \mathrm{H}_{2}$ molecule is energetically next in order as compared to the lowestenergy conformer non-planar dibridged disilyene discussed in the previous section. This structure, denoted as Si-H-SiH, contains one tricenter Si-H-Si bond, while the other hydrogen atom is attached to a $\mathrm{Si}$ atom to form a conventional $\mathrm{Si}-\mathrm{H}$ single bond, with $\mathrm{C}_{\mathrm{s}}$ point group symmetry of the conformer, and a closed-shell electronic ground state of symmetry ${ }^{1} \mathrm{~A}^{\prime}$. Our optimized geometry parameters are (see Fig. 4(b)): (a) two Si-H bond distances in the tricenter Si-H-Si bond are $1.72 \AA$, and $1.63 \AA$, (b) the bond length for the single $\mathrm{Si}-\mathrm{H}$ bond is $1.48 \AA$, and (c) the distance between two silicon atoms is $2.10 \AA$. Inside the Si-H-Si triangle, Si-H-Si bond angle is $77.7^{\circ}$, while the two H-Si-Si bond angles are, $49.2^{\circ}$ and $53.1^{\circ}$. The external H-Si-H bond angle is computed to be $109^{\circ}$, where as the HSi-SiH dihedral angle is $0.6^{\circ}$. Our optimized geometry parameters are in good agreement with the experimental and theoretical results of Cordonnier et al., ${ }^{42}$ and with the theoretical results reported by Colegrove and Schaefer, ${ }^{33}$ Adamczyk and Broadbelt, ${ }^{81}$ Grev and Schaefer, ${ }^{37}$ Sannigrahi and Nandi, ${ }^{38}$ Curtiss et al, ${ }^{45}$ and Koseki and Gordon. ${ }^{82}$ Atomic coordinates corresponding to our optimized ground state geometry of this conformer are presented in Table S10 of the Supporting Information. 
Using these geometry parameters, the calculated photoabsorption spectrum of the mono-bridged conformer is presented in Fig. 6, while detailed information about the excited states contributing to the peaks in the spectrum are presented in Table S2 of the Supporting Information. The optimized geometries of the select excited states corresponding to some of the frontier peaks in the spectrum are given in Fig. S11 of the Supporting Information. The calculated photoabsorption spectrum of this conformer is spread over a wide energy range starting from $2.8 \mathrm{eV}$ to about $9.5 \mathrm{eV}$. The absorption begins with a very feeble peak near $2.81 \mathrm{eV}$, due to an excited state whose wave function largely consists of the $|H \rightarrow L\rangle$ configuration. It is followed by three comparatively stronger peaks near $4.08 \mathrm{eV}, 5.16$ $\mathrm{eV}$, and $5.75 \mathrm{eV}$, all due to excited states whose wave functions are dominated by the singly-excited configurations. The first of these peaks (peak II) derives almost equal intensity from two closely located states exhibiting significant mixing of configurations $|H \rightarrow L+1\rangle,|H-1 \rightarrow L\rangle$, and $|H \rightarrow L+2\rangle$, $|H \rightarrow L+4\rangle$, respectively. The relaxed geometry of this excited state is a disilavinylidene type of structure with the Si-Si and equal Si-H bond length $2.26 \AA$ and $1.48 \AA$, respectively. The optimized geometry of the excited state corresponding to peak III also seems to be a disilavinylidene like structure, with the Si-Si bond length $2.13 \AA$, and equal $\mathrm{Si}-\mathrm{H}$ bond lengths of $1.47 \AA$. The most intense peaks of the spectrum are peaks VIII and IX, located at $7.91 \mathrm{eV}$ and $8.86 \mathrm{eV}$, respectively. Wave functions of the excited states giving rise to these peaks exhibit strong mixing of singly excited configurations. The wave function corresponding to peak VIII is a mixture of characterized of singly-excited configurations $|H-1 \rightarrow L+4\rangle$ and $|H-2 \rightarrow L+2\rangle$, while that corresponding to peak IX is largely composed of $|H-1 \rightarrow L+4\rangle$ and $|H-3 \rightarrow L\rangle$. The relaxed geometry of the excited state corresponding to one of the most intense peaks, i.e., peak VIII, has the elongated Si-Si bond length of almost $2.45 \AA$, and the $\mathrm{H}$-atom of the single $\mathrm{Si}-\mathrm{H}$ bond comes out of the plane with H-Si-Si bond angle $95.5^{\circ}$, as compared to the ground state structure, which is nearly planar. The spectrum terminates with peak X, which appears to be a shoulder of the preceding peak, and is due to an excited state located at $9.16 \mathrm{eV}$, whose wave function derives dominant contributions from the double-excitations $|H \rightarrow L+1 ; H-1 \rightarrow L+2\rangle$ and $|H \rightarrow L+2 ; H \rightarrow L+2\rangle$. When we compare the absorption spectrum of this conformer to that of the lowest-energy structure, we find significant differences. Therefore, it should be possible to distinguish between the two conformers using absorption spectroscopy.

Fig. S2 of the Supporting Information presents the plots of the Hartree-Fock MOs which participate in the photobsorption in the monobridged conformer. 


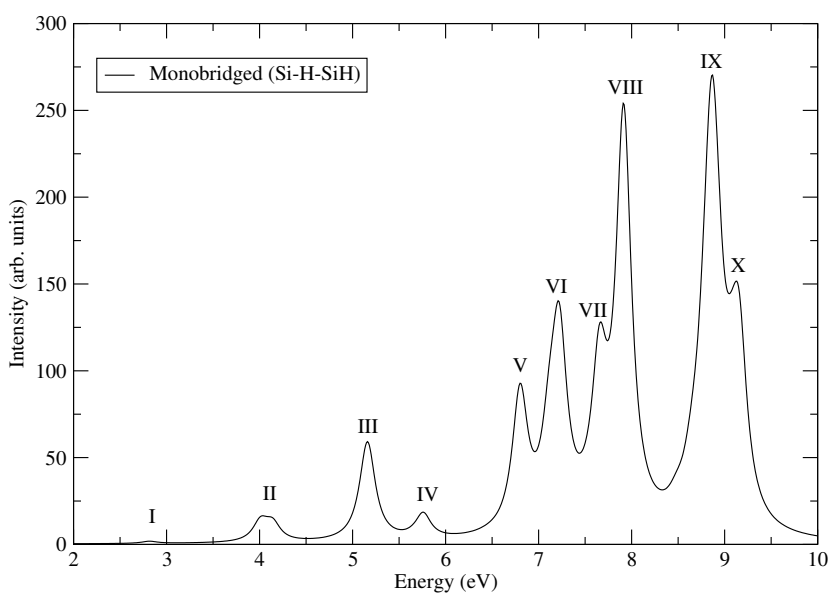

Figure 6: Optical absorption spectrum of monobridged ( $\mathrm{Si}-\mathrm{H}-\mathrm{SiH})$ conformer computed using the MRSDCI method, and cc-pVTZ basis set. For plotting the spectrum, $0.1 \mathrm{eV}$ uniform line-width was assumed.

\section{Disilavinylidene $\left(\mathrm{Si}_{-} \mathrm{SiH}_{2}\right)$}

This conformer is $0.58 \mathrm{eV}$ higher in energy as compared to the lowest $\mathrm{Si}_{2} \mathrm{H}_{2}$ dibridged disilyne structure, but just $0.14 \mathrm{eV}$ above the mono-bridged isomer. Its geometrical configuration is denoted as $\mathrm{Si}^{-\mathrm{SiH}_{2}}$, and has one $\mathrm{Si}$ atom attached to two $\mathrm{H}$ atoms through normal Si-H single bonds, while no $\mathrm{H}$ atoms are attached to the other $\mathrm{Si}$ atom, leading to a $\mathrm{C}_{2 \mathrm{v}}$ symmetric structure (see Fig. 4(c)), and a ground state of symmetry ${ }^{1} \mathrm{~A}_{1}$. Our optimized geometry parameters with the distance between two Si atoms as $2.20 \AA$, both the $\mathrm{Si}-\mathrm{H}$ bonds lengths of $1.48 \AA$, and the $\mathrm{Si}-\mathrm{Si}-\mathrm{H}$ and $\mathrm{H}-\mathrm{Si}-\mathrm{H}$ bond angles as $124^{\circ}$, and $112^{\circ}$, respectively, are in very good agreement with the theoretically reported values by several other groups. ${ }^{33,37,38,45,81,82}$ The atomic coordinates corresponding to our optimized ground state geometry are presented in Table S11 of Supporting information.

The calculated photoabsorption spectrum of the disilavinylidene conformer, corresponding to the optimized geometry, is presented in Fig. 7, while the information related to its peaks such as the oscillator strengths, dominant many particle wave functions of the contributing excited states, their excitation energies etc., are detailed in Table S3 of the Supporting Information. In Fig. S12 of the Supporting Information we present the optimized geometries of some of the excited state contributing to the frontier peaks in the optical absorption spectrum.

The optical absorption spectra of this isomer starts with a tiny peak at $2.48 \mathrm{eV}$, followed by four small peaks near $3.13 \mathrm{eV}, 4.29 \mathrm{eV}, 5.79 \mathrm{eV}$ and $6.43 \mathrm{eV}$, most of which are dominated by the singly-excited configurations, with double excitations also making important contributions in a couple of cases. Wave 
function of the excited state contributing to peak I is dominated by single excitation $|H-1 \rightarrow L\rangle$, while peak II corresponds to a state dominated by the double excitation $|H \rightarrow L ; H \rightarrow L\rangle$. Wave function of the state corresponding to peak III is dominated by singly-excited configuration $|H \rightarrow L+1\rangle$, while the one corresponding to peak IV exhibits significant mixing of the single excitation $|H-2 \rightarrow L\rangle$ and the double excitation $|H \rightarrow L ; H-2 \rightarrow L+1\rangle$. The optimized geometry corresponding to peak III is non-planar, unlike the ground state structure. It has an elongated Si-Si bond with length $2.70 \AA$, and equal Si-H arms, with the bond lengths of $1.52 \AA$ each. The most intense peak of the spectrum (peak VII) occurs at $7.57 \mathrm{eV}$, and is due to a state whose wave function exhibits strong mixing of singly-excited configurations $|H-1 \rightarrow L+2\rangle$ and $|H-1 \rightarrow L+4\rangle$. The optimized structure of this excited state is planar, with the Si-Si bond length $2.24 \AA$, and equal Si-H bond distances of $1.61 \AA$. The last peak of the calculated spectrum (peak IX) is located at $8.51 \mathrm{eV}$, and is due to a state whose wave function consists predominantly of the double excitation $|H \rightarrow L ; H-2 \rightarrow L+1\rangle$. If we compare the absorption spectrum of disilavinylidene conformer to those of the two previously discussed structures, including close-lying monobridged structure, we find significant differences both in terms of peak locations, and relative intensities, thus making their optical detection feasible.

The plots of the orbitals contributing to the absorption spectrum are presented in Fig. S3 of Supporting Information.

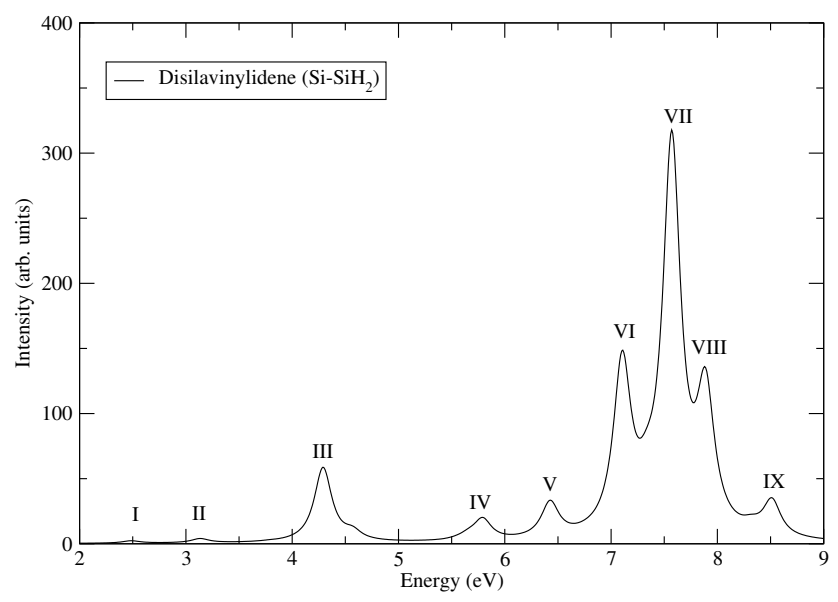

Figure 7: Optical absorption spectrum of disilavinylidene $\left(\mathrm{Si}-\mathrm{SiH}_{2}\right)$ conformer, calculated using the MRSDCI method, and the cc-pVTZ basis set. For plotting the spectrum, $0.1 \mathrm{eV}$ uniform line-width was used. 


\section{Trans-bent (HSi-SiH)}

The trans-bent conformer, which has the highest energy as compared to rest of the three conformers considered in this work, has the geometrical configuration denoted as $\mathrm{HSi}-\mathrm{SiH}$. This structure is just $0.22 \mathrm{eV}$ higher in energy as compared to the disilavinylidene conformer, and with two Si-H single bonds has the point group symmetry $C_{2 h}$, as shown in Fig. 4(d). Our optimized geometry parameters with $\mathrm{Si}-\mathrm{H}$ and $\mathrm{Si}-\mathrm{Si}$ bond distances of $1.49 \AA$, and $2.10 \AA$, respectively, and the $\mathrm{Si}-\mathrm{Si}-\mathrm{H}$ bond angle of $125.6^{\circ}$, are in good agreement with those reported by Adamczyk and Broadbelt ${ }^{81}$ computed using the G3//B3LYP approach, and by Sannigrahi and Nandi ${ }^{38}$ based on ab initio SCF calculations. Using a DFT based approach, employing various basis sets, and exchange-correlation functionals, Jursic obtained the optimized bond lengths of the trans-bent structure, which are also in good agreement with our results. ${ }^{39}$ The atomic coordinates corresponding to our optimized ground state geomery of this structure are presented in Table S12 of Supporting Information.

Using our optimized geometry, the photoabsorption spectrum of the trans-bent conformer computed using the MRSDCI approach, from its ${ }^{1} A_{g}$ ground state, is presented in Fig. 8, while the detailed information pertaining to the excited states contributing to various peaks is given in Table S4 of Supporting Information. The optimized geometries of a few excited states, corresponding to some important peaks in absorption spectrum of this isomer, are presented in Fig. S13 of the Supporting Information. Absorption in the trans-bent conformer starts with a feeble peak at $1.16 \mathrm{eV}$, due to a state whose wave function is dominated by the singly-excited configuration $|H \rightarrow L\rangle$, and the relaxed geometry of this state, unlike the ground state is neither trans-like, nor planar. The H-atom on one side is out of the plane, but both the Si-H bond length are still equal (1.49 $\AA$ ), whereas the Si-Si bond length is $2.12 \AA$. This is followed by the most intense peak of the spectrum at $2.96 \mathrm{eV}$, due to a state whose wave function exhibits strong mixing of singly-excited configurations $|H-1 \rightarrow L\rangle$ and $|H \rightarrow L+1\rangle$. We note that the location of the most intense peak at a much lower energy, as compared to the other three conformers, is enough of a distinguishing feature of this conformer to allow its detection through optical spectroscopy. However, the relaxed geometry corresponding to this peak (peak II), is almost similar to the ground state structure, with the $\mathrm{Si}-\mathrm{H}$ and $\mathrm{Si}-\mathrm{Si}$ bond distances of $1.52 \AA$, and $2.33 \AA$, respectively. It is followed by two relatively weaker features III and IV at $4.56 \mathrm{eV}$, and $5.05 \mathrm{eV}$, respectively. The wave functions of the state corresponding to peak III is dominated by the single excitation $|H-1 \rightarrow L+1\rangle$, while 
that of peak IV exhibits strong mixing of singly-excited configurations $|H \rightarrow L+1\rangle$ and $|H-1 \rightarrow L\rangle$. Next three features V, VI, and VII located at $6.00 \mathrm{eV}, 6.28 \mathrm{eV}$, and $6.59 \mathrm{eV}$, respectively, are due to states whose wave functions derive strong contributions from doubly-excited configurations. Feature V, which is a shoulder to peak VI is due to a state whose wave function is largely composed of the double excitation $|H \rightarrow L ; H \rightarrow L+2\rangle$, while VI and VII exhibit strong mixing of double and single excitations $|H-1 \rightarrow L ; H \rightarrow L+2\rangle$, and $|H \rightarrow L+3\rangle$. The computed spectrum has its last peak at $7.35 \mathrm{eV}$ due to two close lying states at $7.32 \mathrm{eV}$ and $7.42 \mathrm{eV}$, which exhibit strong mixing of double excitations $|H-2 \rightarrow L ; H \rightarrow L\rangle,|H \rightarrow L+2 ; H \rightarrow L+1\rangle$, and $|H-2 \rightarrow L ; H-1 \rightarrow L\rangle,|H-2 \rightarrow L+1 ; H \rightarrow L\rangle$, respectively.

In Fig. S4 of Supporting Information, the plots of the frontier orbitals participating in the optical absorption in this conformer are presented.

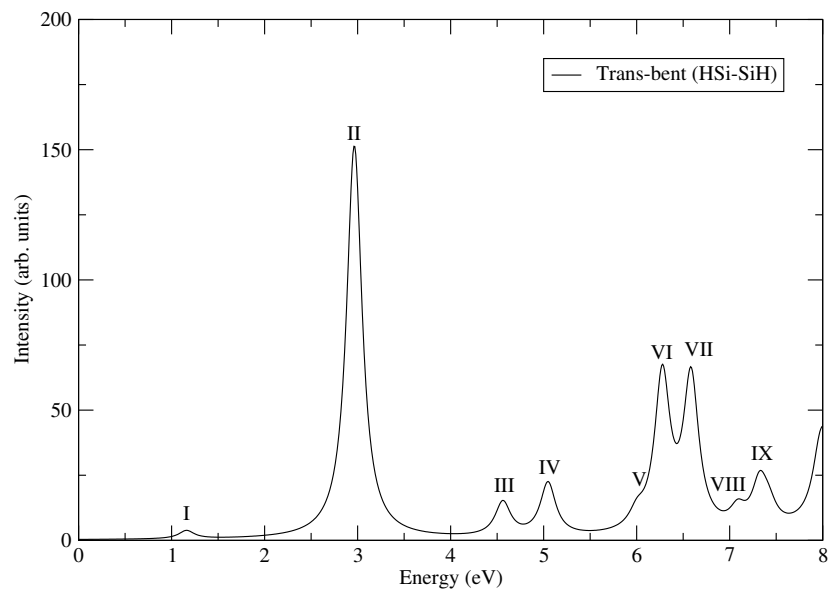

Figure 8: Optical absorption spectrum of trans-bent (HSi-SiH) conformer computed using the MRSDCI method and cc-pVTZ basis set. For plotting the spectrum, a uniform line-width of $0.1 \mathrm{eV}$ was used.

\section{$\mathrm{Si}_{2} \mathrm{H}_{4}$}

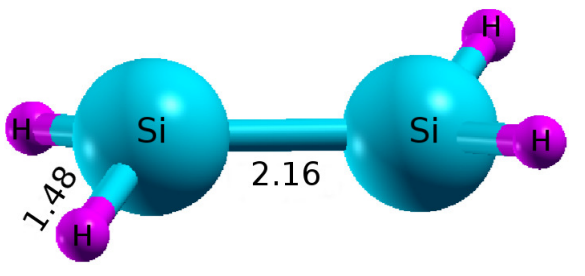

(a) Disilene

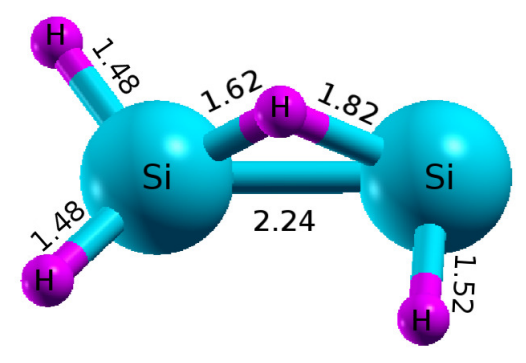

(b) Monobridged

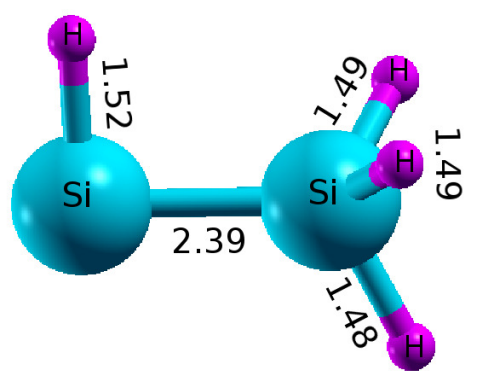

(c) Silylsilylene

Figure 9: Ground state geometries of $\mathrm{Si}_{2} \mathrm{H}_{4}$ isomers, optimized using the CCSD(T) method, and the cc-pVTZ basis set. All the bond lengths are in $\AA$ unit. 
Geometries and relative stabilities of the isomers of $\mathrm{Si}_{2} \mathrm{H}_{4}$ have been studied extensively over the years using electronic-structure methods by several authors. ${ }^{26,28-30,34,35,38,40,45-48}$ Earlier, two main isomers of $\mathrm{Si}_{2} \mathrm{H}_{4}$ were investigated, namely, disilene containing of a $\mathrm{Si}-\mathrm{Si}$ double bond, and silylsilylene, a completely singly-bonded structure. ${ }^{34,35}$ For disilene as well several structures are possible, but two main structures have been investigated, namely: (a) planar ethylene like structure consisting of a true Si-Si double bond, with $D_{2 h}$ symmetry, and (b) a trans-bent structure with a nominal Si-Si double bond, with $C_{2 h}$ symmetry. ${ }^{30,34,38,46,47}$ However, it was soon revealed by calculations of vibrational frequencies, that planar disilene was not stable, and actually corresponded to a transition state on the potential energy surface. ${ }^{34,46,47}$ Later on a new low-lying isomer with a monobridged structure was discovered computationally, and found to be stable. ${ }^{26,41,48}$ The present-day consensus is that the trans-bent disilene structure is energetically the lowest, followed by mono-bridged, and silylsilylene isomers, lying slightly above it. ${ }^{26,40,41,48}$. We performed our own geometry optimization for these three structures using the $\operatorname{CCSD}(\mathrm{T})$ method, coupled with the cc-pVTZ basis, and the optimized geometries are shown in Fig. 9. Energetically speaking, we found the ordering trans-bent $<$ mono-bridged $<$ silylsilylene, with mono-bridged and silylsilylene structures being almost degenerate and just $0.286 \mathrm{eV}$ and $0.294 \mathrm{eV}$ above the trans-bent structure (see Table 1). Thus, our results on the geometries and energies of these isomers are in excellent agreement with the results of recent calculations. ${ }^{26,40,41,48}$ Next, we discuss the geometries and optical absorption spectra of the individual isomers in the following sections.

\section{Disilene $\left(\mathrm{H}_{2} \mathrm{Si}_{\text {-SiH }}\right)$}

The trans-bent structure of disilene, with $\mathrm{C}_{2 \mathrm{~h}}$ point group symmetry, has the lowest energy among all the $\mathrm{Si}_{2} \mathrm{H}_{4}$ isomers. The optimized geometry of this conformer has equal bond lengths of $1.48 \AA$ for the four Si-H bonds. Additionally, Si-Si bond length was computed to be $2.16 \AA$, along with the HSi-H, and H-Si-Si bond angles of $113^{\circ}$, and $119^{\circ}$, respectively, whereas the HSi-SiH dihedral angle is $33^{\circ}$ (see Fig. 9(a)). These values are in good agreement with the geometry parameters reported in the

literature. ${ }^{26,28,30,40,41}$ The atomic coordinates corresponding to our optimized ground state geometry of this conformer are presented in Table S13 of Supporting information.

The calculated photoabsorption spectrum of the trans-bent disilene $\left(\mathrm{H}_{2} \mathrm{Si}_{-} \mathrm{SiH}_{2}\right)$ conformer is presented in Fig. 10, and the corresponding dominant many particle wave functions contributing to the peaks of the optical spectra are given in Table S5 of the Supporting Information. The relaxed excited 
state geometries corresponding to some of the frontier peaks of optical absorption spectrum of this isomer is given in Fig. S14 of the Supporting Information.

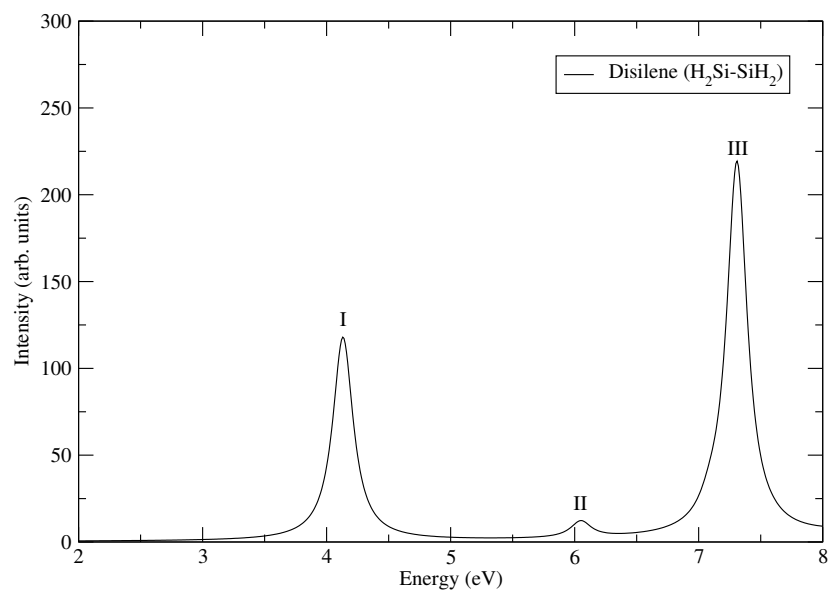

Figure 10: Optical absorption spectrum of disilene $\left(\mathrm{H}_{2} \mathrm{Si}_{-} \mathrm{SiH}_{2}\right)$ conformer computed using the MRSDCI method, along with cc-pVTZ basis set. For plotting the spectrum, a uniform line-width of $0.1 \mathrm{eV}$ was assumed.

The optical absorption spectrum of this disilene conformer starts with a intense peak at $4.13 \mathrm{eV}$, corresponding to an excited state whose wave function is dominated by singly-excited configuration $|H \rightarrow L\rangle$. As far as the relaxed geometry of this state is concerned, as compared to the ground state SiSi bond length becomes longer, around $2.49 \AA$, whereas the $\mathrm{Si}-\mathrm{H}$ bond length does not change too much $(1.49 \AA)$. But the dihedral angle (H-Si-Si-H) increases from $33^{\circ}$ to $57^{\circ}$ as compared to the ground state structure. It is followed by a weak peak at $6.05 \mathrm{eV}$, due to an excited state with wave function deriving dominant contributions from the singly-excited configurations $|H \rightarrow L+3\rangle$ and $|H \rightarrow L+4\rangle$. The most intense peak, with a very large oscillator strength, is located at $7.31 \mathrm{eV}$, with many-particle wave function dominated again by same set of single excitations $|H \rightarrow L+3\rangle$ and $|H \rightarrow L+4\rangle$. In contrast to the ground state, the optimized geometry of this state (peak III) is completely planar with the $\mathrm{Si}-\mathrm{Si}$ bond length $2.16 \AA$, and equal Si-H bond distances of $1.47 \AA$. In Fig. S5 of Supporting Information, the plots of the frontier orbitals participating in the optical absorption in disilene are presented.

\section{Monobridged $\left(\mathrm{H}_{2} \mathrm{Si}-\mathrm{H}-\mathrm{SiH}\right)$}

The ground state of monobridged conformer has $\mathrm{C}_{1}$ symmetry, and is predicted to be only $0.286 \mathrm{eV}$ (see Table 1) higher than the ground state of disilene conformer of $\mathrm{Si}_{2} \mathrm{H}_{4}$. Because of this close energetic proximity of the monobridged isomer to disilene, their optical absorption spectra become important because they offer the possibility of optical identification of these close-lying isomers. Schaefer and co- 
workers $^{26}$, by means of highly-correlated ab initio calculations, were the first ones to predict this isomer. Later on, the same group ${ }^{41}$ detected this isomer experimentally using Fourier transform microwave spectroscopy technique. Our optimized geometry obtained using the CCSD(T) method, and the ccpVTZ basis set, is shown in Fig. 9(b). The optimized lengths were found to be $2.24 \AA$ for Si-Si bond, 1.48 $\AA$ for the two Si-H bonds on the left, and $1.52 \AA$ for the Si-H bond on the right. On the left side, the three $\mathrm{H}-\mathrm{Si}-\mathrm{H}$ bond angles are $103.1^{\circ}, 106.8^{\circ}$ and $108.3^{\circ}$, while on the right, the H-Si-Si bond angle is 85. $4^{\circ}$. For the bridged hydrogen atom, our optimized Si-H bond lengths are $1.62 \AA$, and $1.82 \AA$. Inside the Si-H-Si triangle, the Si-H-Si bond angle is $81.4^{\circ}$, while the two H-Si-Si bond angles are $53.2^{\circ}$, and $45.4^{\circ}$, respectively, from left to right. Additionally, H-Si-H bond angle on the right is optimized to be 84.3 $3^{\circ}$. All the HSi-SiH dihedral angles with the bridged $\mathrm{H}$-atom are near about $85^{\circ}$. These results are in good agreement with the values reported by Schaefer and coworkers. ${ }^{26,41}$ The atomic coordinates corresponding to our optimized ground state geometry of the monobridged conformer are presented in Table S14 of Supporting information.

The calculated photoabsorption spectrum of the monobridged $\left(\mathrm{H}_{2} \mathrm{Si}-\mathrm{H}-\mathrm{SiH}\right)$ conformer, using our optimized geometry, is presented in Fig. 11, while the detailed information about the excited states contributing to various peaks, including their many particle wave functions are presented in Table S6 of the Supporting Information. The optimized geometries of excited states contributing to some of the frontier peaks of optical absorption spectrum are presented in Fig. S15 of the Supporting Information.

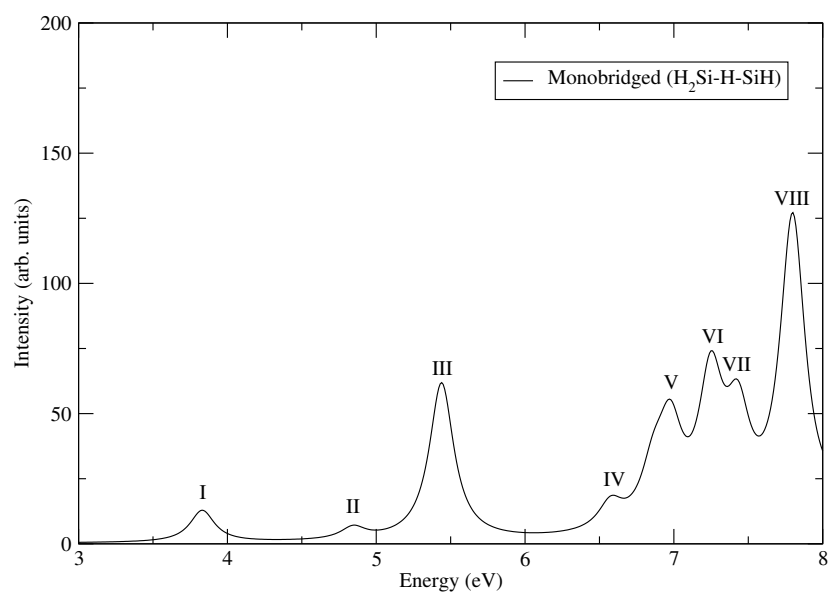

Figure 11: Optical absorption spectrum of monobridged $\left(\mathrm{H}_{2} \mathrm{Si}-\mathrm{H}-\mathrm{SiH}\right)$ conformer computed using the MRSDCI method, and cc-pVTZ basis set. For plotting the spectrum, a uniform line-width of $0.1 \mathrm{eV}$ was assumed.

As compared to the disilene conformer, optical photoabsorption spectrum of the monobridged isomer consists of several well-separated peaks over the same energy range. It starts with a small peaks at 3.83 
$\mathrm{eV}$, due to a state whose many-particle wave function is dominated by the singly-excited configuration $|H \rightarrow L\rangle$. In the relaxed geometry of this excited state, the Si-Si bond length $(2.36 \AA)$ is elongated a bit more compared to the ground state. Furthermore, the geometry is qualitatively different as compared to the ground state, with the bridged H-atom is now connected with just one Si-atom. Consequently, one Si-atom is connected with three H atoms, while the other one is connected to just one, like silylsilylene but with all $\mathrm{Si}-\mathrm{H}$ bond lengths being close to $1.48 \AA$. This is followed by an even weaker peak at $4.84 \mathrm{eV}$, due to a state whose wave function derives its main contribution from the configuration $|H-1 \rightarrow L\rangle$, and whose optimized geometry is also similar to a silylsilylene-type structure. The Si-Si bond length is 2.45 $\AA$, but with equal Si-H bond lengths on the end where three H atoms are bonded. However, the Si-H bond where the Si-atom is bonded to only one H-atom, is comparatively longer $(1.60 \AA)$. A comparatively intense peak appears at $5.44 \mathrm{eV}$, with the wave function of the excited state dominated by the singlyexcited configuration $|H \rightarrow L+1\rangle$. The relaxed geometry of this excited state is almost similar to that of the first one, with same Si-Si and Si-H bond lengths, but with totally different HSi-SiH dihedral angles. This is followed by a series of five well-separated peaks ranging from $6.58 \mathrm{eV}$ to the most intense peak (peak VIII) of the spectrum located at $7.80 \mathrm{eV}$. Various properties of the excited states, along with their wave functions for this conformer are presented in Table S6 of the Supporting Information, from where it is obvious that the wave functions of all the states are dominated by singly-excited configurations, except for peak VII whose wave function is dominated by a configuration consisting of HOMO-LUMO double excitation $|H \rightarrow L ; H \rightarrow L\rangle$. The wave function of the excited state giving rise to the most intense peak VIII, is dominated by single excitations $|H \rightarrow L+4\rangle$, and $|H-3 \rightarrow L\rangle$. If we compare the absorption spectrum of the monobridged isomer with that of disilene, we note that they are sufficiently different both in terms of the number of peaks, and their locations, such that the optical spectroscopy can be used for their identification. The frontier MOs participating in various optical excitations of this conformer are presented in Fig. S6 of the Supporting Information.

\section{Silylsilylene $\left(\mathrm{H}_{3} \mathrm{Si}-\mathrm{SiH}\right)$}

Similar to the case of disilene, over the years extensive research has been done on the electronic structure and geometry of silylsilylene $\left(\mathrm{H}_{3} \mathrm{Si}-\mathrm{SiH}\right)$ conformer of $\mathrm{Si}_{2} \mathrm{H}_{4} \cdot{ }^{41,81}$ Our optimized geometrical parameters of its ground state of $\mathrm{C}_{\mathrm{s}}$ symmetry, obtained using the $\operatorname{CCSD}(\mathrm{T})$ approach, and cc-pVTZ basis set are:

(a) Si-Si bond length $2.39 \AA$, and (b) Si-H bond lengths of $1.49 \AA, 1.49 \AA$, and $1.48 \AA$ on the right side, 
with $\mathrm{H}-\mathrm{Si}-\mathrm{H}$ bond angles $109.5^{\circ}, 109.5^{\circ}$ and $113.9^{\circ}$. On the left side optimized Si-H bond length is 1.52 $\AA$, along with the H-Si-Si bond angle $89.2^{\circ}$, whereas the HSi-SiH dihedral angles are 59.2 $2^{\circ}, 59.2^{\circ}$, and $180^{\circ}$, respectively (see Fig. 9(c)). These values are in good agreement with those reported by McCarthy et al, ${ }^{41}$ and Adamczyk et al. ${ }^{81}$ The atomic coordinates corresponding to our optimized ground state geometry of this conformer are presented in Table S15 of Supporting information.

Energetically speaking, silysilylene is just $0.294 \mathrm{eV}$ higher than disilene, and a negligible $0.008 \mathrm{eV}$ higher than the monobridged isomer. Again, the question arises, whether their optical absorption spectra are sufficiently different so as to allow their identification using this spectroscopy.

Using our optimized geometry, we present the calculated photoabsorption spectrum of silylsilylene in Fig. 12, and the optimized excited state geometries corresponding to some of the peaks of optical absorption spectrum of this isomer are given in Fig. S16 of the Supporting Information. The most noteworthy point is that this confomer, except for a couple of very feeble peaks, does not exhibit any significant absorption till about $6 \mathrm{eV}$, beyond which the intensity of the absorption rises. This aspect of its absorption spectrum distinguishes it from those of the two previous conformers, and can be used as a fingerprint for its optical detection.

The absorption spectrum of silylsilene conformer starts with very weak peaks located at $1.93 \mathrm{eV}$, and $3.79 \mathrm{eV}$, due to excited states whose many-particle wave functions are dominated by the singly-excited configurations $|H \rightarrow L\rangle$, and $|H-1 \rightarrow L\rangle$, respectively. The optimized geometry corresponding to peak I has all Si-H bond lengths are close to $1.48 \AA$, whereas the $\mathrm{Si}-\mathrm{Si}$ bond length is reduced to $2.36 \AA$. The most intense peak (peak V) appears at $6.97 \mathrm{eV}$, due to a state whose wave functions are dominated by single excitations $|H \rightarrow L+1\rangle$, and $|H \rightarrow L+2\rangle$. In the relaxed geometry of the excited state corresponding to the most intense peak (peak V), all the Si-H bond lengths of the right side are $1.48 \AA$, but the Si-Si bond length, and the Si-H bond distance of the left side are elongated to $3.06 \AA$, and 1.56 $\AA$, respectively. The last peak of our calculated spectrum (peak VI) is located at $7.35 \mathrm{eV}$, and is due to a state with wave function deriving main contribution from $|H \rightarrow L+3\rangle$ configuration, with a smaller contribution from the single excitation $|H-1 \rightarrow L+1\rangle$. Detailed information about all the excited states contributing to various peaks in the absorption spectrum of silylsilylene is presented in Table S7 of the Supporting Information, whose Fig. S7 contains plots of MOs participating in the absorption. 


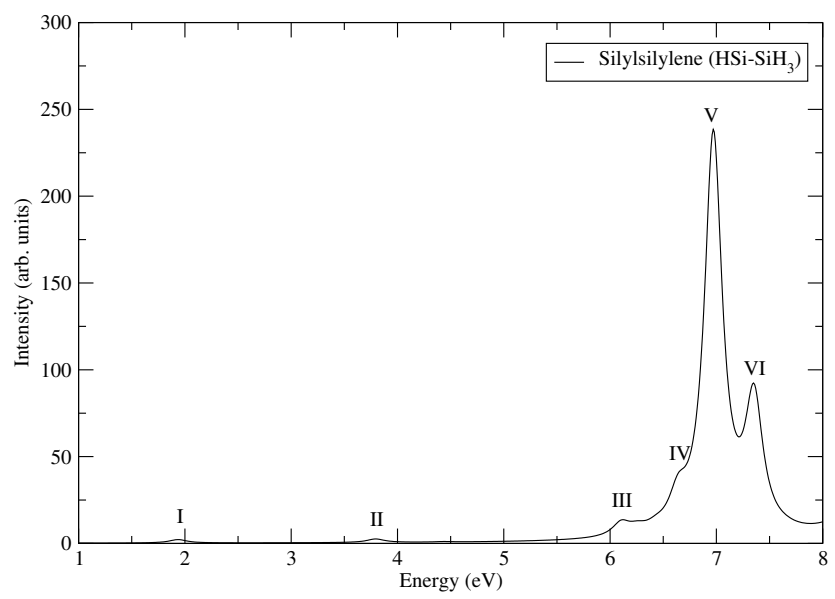

Figure 12: Optical absorption spectrum of silylsilylene $\left(\mathrm{H}_{3} \mathrm{Si}-\mathrm{SiH}\right)$ conformer computed using the MRSDCI method, and cc-pVTZ basis set. For plotting the spectrum, a uniform line-width of $0.1 \mathrm{eV}$ was used.

\section{Conclusions}

In this work we presented state of the art quantum chemical calculations, utilizing large basis sets, aimed at obtaining electronic structure, geometrical, and optical properties of hydrides of silicon dimer of the class $\mathrm{Si}_{2} \mathrm{H}_{2 n}, n=1,2,3$. Geometry optimization was carried out using the CCSD(T) approach, while the optical absorption spectra was computed by means of large-scale MRSDCI calculations. For $\mathrm{Si}_{2} \mathrm{H}_{2}$ and $\mathrm{Si}_{2} \mathrm{H}_{4}$ several stable, energetically low-lying, and very closely spaced, conformers were considered, and it was discovered that each conformer has a distinct optical absorption spectrum, suggesting their possible detection and identification using absorption spectroscopy. Although, no experimental results exist on the absorption spectra of $\mathrm{Si}_{2} \mathrm{H}_{2}$ and $\mathrm{Si}_{2} \mathrm{H}_{4}$ conformers, but for disilane $\left(\mathrm{Si}_{2} \mathrm{H}_{6}\right)$ excellent agreement with experimental measurement was found. This suggests that our computational methodology based on the MRSDCI method is sound, and, therefore, our calculated spectra for $\mathrm{Si}_{2} \mathrm{H}_{2}$ and $\mathrm{Si}_{2} \mathrm{H}_{4}$ conformers must be trustworthy.

Furthermore, we also obtained the relaxed geometries of several important optically excited states of various clusters, and found that optical absorption leads to significant geometry changes in these clusters, as compared to their ground state. We hope that there will be future experimental efforts to measure the absorption spectra of conformers of $\mathrm{Si}_{2} \mathrm{H}_{2}$ and $\mathrm{Si}_{2} \mathrm{H}_{4}$, against which our results could be benchmarked. 


\section{Supporting Information}

The supporting information consists of a file containing important information about the excited states contributing to peaks in our computed absorption spectra of various conformers of $\mathrm{Si}_{2} \mathrm{H}_{2}, \mathrm{Si}_{2} \mathrm{H}_{4}$, and $\mathrm{Si}_{2} \mathrm{H}_{6}$. It consists of plots of frontier molecular orbitals, excitation energies, oscillator strengths, dominant terms in their many-body wave functions, polarization directions of absorbed photons, and the excited state geometries corresponding to some of the peaks of optical absorption spectra.

\section{Author Information}

\section{Corresponding Authors}

Alok Shukla: *E-mail: shukla@phy.iitb.ac.in

\section{Notes}

The authors declare no competing financial interests.

\section{Acknowledgements}

Work of P.B. was supported by a Senior Research Fellowship offered by University Grants Commission, India.

\section{TOC Graphic}

\section{Linear Optical Absorption of $\mathrm{Si}_{2} \mathrm{H}_{4}$ Isomers}
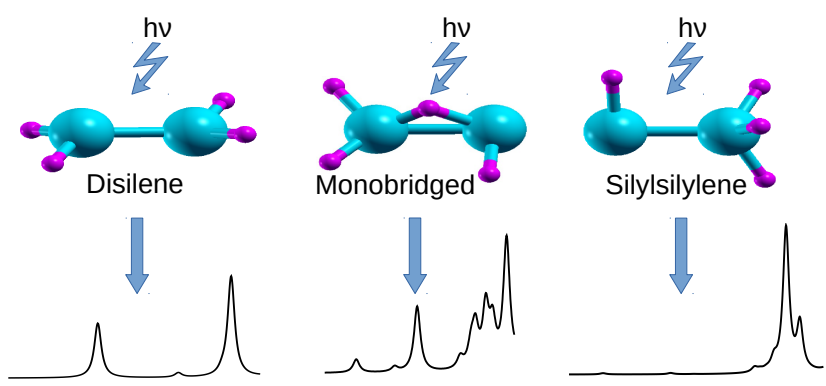


\section{References}

(1) Zhao, J.; Liu, H.; Yu, Z.; Quhe, R.; Zhou, S.; Wang, Y.; Liu, C. C.; Zhong, H.; Han, N.; Lu, J.; Yao, Y.; Wu, K. Rise of silicene: A competitive 2D material. Progress in Materials Science 2016, $83,24-151$.

(2) Vogt, P.; De Padova, P.; Quaresima, C.; Avila, J.; Frantzeskakis, E.; Asensio, M. C.; Resta, A.; Ealet, B.; Le Lay, G. Silicene: Compelling Experimental Evidence for Graphenelike TwoDimensional Silicon. Phys. Rev. Lett. 2012, 108, 155501.

(3) Ni, Z.; Liu, Q.; Tang, K.; Zheng, J.; Zhou, J.; Qin, R.; Gao, Z.; Yu, D.; Lu, J. Tunable Bandgap in Silicene and Germanene. Nano Letters 2012, 12, 113-118, PMID: 22050667.

(4) Jose, D.; Datta, A. Structures and Chemical Properties of Silicene: Unlike Graphene. Accounts of Chemical Research 2014, 47, 593-602, PMID: 24215179.

(5) Kingon, A. I.; Maria, J.-P.; Streiffer, S. K. Alternative dielectrics to silicon dioxide for memory and logic devices. Nature 2000, 406, 1032-1038.

(6) Thomson, D. et al. Roadmap on silicon photonics. Journal of Optics 2016, 18, 073003.

(7) Aberle, A. G. Surface passivation of crystalline silicon solar cells: a review. Progress in Photovoltaics: Research and Applications 2000, 8, 473-487.

(8) Stefaan, D. W.; Antoine, D.; C., H. Z.; Christophe, B. High-efficiency Silicon Heterojunction Solar Cells: A Review. green 2012, 2, 7-24.

(9) Tiedje, T.; Cebulka, J. M.; Morel, D. L.; Abeles, B. Evidence for Exponential Band Tails in Amorphous Silicon Hydride. Phys. Rev. Lett. 1981, 46, 1425-1428.

(10) Tsai, C.; Li, K.; Kinosky, D. S.; Qian, R.; Hsu, T.; Irby, J. T.; Banerjee, S. K.; Tasch, A. F.; Campbell, J. C.; Hance, B. K.; White, J. M. Correlation between silicon hydride species and the photoluminescence intensity of porous silicon. Applied Physics Letters 1992, 60, 1700-1702.

(11) Cody, G.; Wronski, C.; Abeles, B.; Stephens, R.; Brooks, B. Optical characterization of amorphous silicon hydride films. Solar Cells 1980, 2, $227-243$. 
(12) Gates, S. M.; Kunz, R. R.; Greenlief, C. Silicon hydride etch products from the reaction of atomic hydrogen with Si(100). Surface Science 1989, 207, $364-384$.

(13) Swihart, M. T.; Girshick, S. L. Thermochemistry and Kinetics of Silicon Hydride Cluster Formation during Thermal Decomposition of Silane. The Journal of Physical Chemistry B 1999, 103, 64-76.

(14) Ring, M. A.; O’Neal, H. E. Mechanism of the thermally induced gas-phase decomposition of silane: a revisitation. The Journal of Physical Chemistry 1992, 96, 10848-10855.

(15) Collins, R.; Ferlauto, A. Advances in plasma-enhanced chemical vapor deposition of silicon films at low temperatures. Current Opinion in Solid State and Materials Science 2002, 6, 425 - 437.

(16) Kenichi Tonokura, T. M.; Koshi, M. Formation Mechanism of Hydrogenated Silicon Clusters during Thermal Decomposition of Disilane. The Journal of Physical Chemistry B 2002, 106, 555-563.

(17) Stuckelberger, M.; Biron, R.; Wyrsch, N.; Haug, F.-J.; Ballif, C. Review: Progress in solar cells from hydrogenated amorphous silicon. Renewable and Sustainable Energy Reviews 2017, 76, 1497 $-1523$.

(18) Wei, W.; Dai, Y.; Huang, B. Hydrogenation of silicene on $\operatorname{Ag}(111)$ and formation of half-silicane. J. Mater. Chem. A 2017, 5, 18128-18137.

(19) Medina, D. B.; Salomon, E.; Lay, G. L.; Angot, T. Hydrogenation of silicene films grown on $\mathrm{Ag}(111)$. Journal of Electron Spectroscopy and Related Phenomena 2017, 219, 57 - 62, SI: The electronic structure of $2 \mathrm{D}$ and layered materials.

(20) Mironov, V. F.; Nepomnina, V. V. The synthesis of alkenylsilanes by the high-temperature condensation of unsaturated compounds with silicon hydrides. Bulletin of the Academy of Sciences of the USSR, Division of chemical science 1961, 10, 1759-1761.

(21) Speier, J. L.; Webster, J. A.; Barnes, G. H. The Addition of Silicon Hydrides to Olefinic Double Bonds. Part II. The Use of Group VIII Metal Catalysts. Journal of the American Chemical Society 1957, 79, 974-979. 
(22) Andrews, L.; Wang, X. Infrared Spectra of the Novel Si2H2 and Si2H4 Species and the SiH1,2,3 Intermediates in Solid Neon, Argon, and Deuterium. The Journal of Physical Chemistry A 2002, $106,7696-7702$.

(23) B., R.; J., B. Photoionization mass spectrometric studies of the transient species $\operatorname{Si} 2 \mathrm{Hn}(\mathrm{n}=2-5)$. The Journal of Chemical Physics 1991, 95, 2416-2432.

(24) C., B. M. B. H. D.; L., D. J. Nonclassical double-bridged structure in silicon-containing molecules: Experimental evidence in $\mathrm{Si}_{2} \mathrm{H}_{2}$ from its submillimeter-wave spectrum. Phys. Rev. Lett. 1991, 66, 413-416.

(25) L., B. M. B. H. C. M. D. C. D. J.; G., C. A. Millimeter- and submillimeter-wave spectroscopy of dibridged Si2H2 isotopomers: Experimental and theoretical structure. The Journal of Chemical Physics 1994, 100, 8614-8624.

(26) III, L. S. M. C. M. H. F. S.; Thaddeus, P. Mono- and Dibridged Isomers of Si2H3 and Si2H4: the True Ground State Global Minima. Theory and Experiment in Concert. Journal of the American Chemical Society 2003, 125, 11409-11417, PMID: 16220964.

(27) Mohapatra, C.; Kundu, S.; Paesch, A. N.; Herbst-Irmer, R.; Stalke, D.; Andrada, D. M.; Frenking, G.; Roesky, H. W. The Structure of the Carbene Stabilized Si2H2 May Be Equally Well Described with Coordinate Bonds as with Classical Double Bonds. Journal of the American Chemical Society 2016, 138, 10429-10432, PMID: 27494691.

(28) Chaeho Pak, J. C. R.-K.; III, H. F. S. Electron Affinities of Silicon Hydrides: SiHn $(n=0-4)$ and Si2Hn (n = 0-6). The Journal of Physical Chemistry A 2000, 104, 11232-11242.

(29) Sax, A. F.; Kalcher, J. Theoretical enthalpies of formation for small silicon hydrides. The Journal of Physical Chemistry 1991, 95, 1768-1783.

(30) Sax, A. F. Pseudopotential calculations on Si2H6 and Si2H4. Journal of Computational Chemistry $1985,6,469-477$.

(31) K., S.; D., A. R.; C., H. N. Disilene, silylsilylene and their cations. Theoretica Chimica Acta 1986, $70,393-406$. 
(32) Poirier, R. A.; Goddard, J. D. The isomers of Si2H4: disilene and silylsilylene. Chemical Physics Letters 1981, 80, $37-41$.

(33) Colegrove, B. T.; Schaefer, H. F. I. Disilyne (Si2H2) revisited. The Journal of Physical Chemistry 1990, 94, 5593-5602.

(34) Lischka, H.; Kohler, H.-J. On the structure and stability of singlet and triplet disilene and silylsilylene. Chemical Physics Letters 1982, 85, 467 - 471.

(35) Koehler, H. J.; Lischka, H. A systematic investigation on the structure and stability of the lowest singlet and triplet states of $\mathrm{Si} 2 \mathrm{H} 4$ and $\mathrm{SiH} 3 \mathrm{SiH}$ and the carbon analogous compounds $\mathrm{SiH} 2 \mathrm{CH} 2$, $\mathrm{SiH} 3 \mathrm{CH}, \mathrm{CH} 3 \mathrm{SiH}, \mathrm{C} 2 \mathrm{H} 4$, and CH3CH. Journal of the American Chemical Society 1982, 104, $5884-5889$.

(36) Lischka, H.; Koehler, H. J. Ab initio investigation on the lowest singlet and triplet state of disilyne (Si2H2). Journal of the American Chemical Society 1983, 105, 6646-6649.

(37) S., G. R.; F., S. H. The remarkable monobridged structure of Si2H2. The Journal of Chemical Physics 1992, 97, 7990-7998.

(38) Sannigrahi, A.; Nandi, P. Ab initio SCF study of the nature of bonding in Si2H2 and Si2H4. Chemical Physics Letters 1992, 188, 575 - 583.

(39) Jursic, B. S. Density functional theory investigation of the Si2H2 nonclassical and tetrahedron distorted structures. Journal of Molecular Structure: $\{$ THEOCHEM\} 1999, 491, $1-9$.

(40) Olbrich, G. On the structure and stability of Si2H4. Chemical Physics Letters 1986, 130, 115 119.

(41) McCarthy M. C.; Yu Z., S. L. S. H. F.; P., T. Monobridged Si2H4. The Journal of Chemical Physics 2006, 124, 074303.

(42) Cordonnier, M.; Bogey, M.; Demuynck, C.; Destombes, J.-L. Nonclassical structures in siliconcontaining molecules: The monobridged isomer of Si2H2. The Journal of Chemical Physics 1992, 97, 7984-7989. 
(43) Raabe, G.; Michl, J. Multiple bonding to silicon. Chemical Reviews 1985, 85, 419-509.

(44) WEST, R.; FINK, M. J.; MICHL, J. Tetramesityldisilene, a Stable Compound Containing a SiliconSilicon Double Bond. Science 1981, 214, 1343-1344.

(45) Curtiss, L. A.; Raghavachari, K.; Deutsch, P. W.; Pople, J. A. Theoretical study of Si2Hn (n=0-6) and $\mathrm{Si} 2 \mathrm{H}+\mathrm{n}(\mathrm{n}=0-7)$ : Appearance potentials, ionization potentials, and enthalpies of formation. The Journal of Chemical Physics 1991, 95, 2433-2444.

(46) Trinquier, G. Double bonds and bridged structures in the heavier analogs of ethylene. Journal of the American Chemical Society 1990, 112, 2130-2137.

(47) Krogh-Jespersen, K. Geometries and relative energies of singlet silylsilylene and singlet disilene. The Journal of Physical Chemistry 1982, 86, 1492-1495.

(48) Dolgonos, G. Relative stability and thermodynamic properties of Si2H4 isomers. Chemical Physics Letters 2008, 466, $11-15$.

(49) Gutowsky, H. S.; Stejskal, E. O. The Infrared Spectrum of Disilane. The Journal of Chemical Physics 1954, 22, 939-943.

(50) Itoh, U.; Toyoshima, Y.; Onuki, H.; Washida, N.; Ibuki, T. Vacuum ultraviolet absorption cross sections of SiH4, GeH4, Si2H6, and Si3H8. The Journal of Chemical Physics 1986, 85, 4867-4872.

(51) Bock, H.; Ensslin, W.; Feher, F.; Freund, R. Photoelectron spectra and molecular properties. LI. Ionization potentials of silanes SinH2n+2. Journal of the American Chemical Society 1976, 98, $668-674$.

(52) EnBlin, W.; Bergmann, H.; Elbel, S. Photoelectron spectra of polysilanes. Conformational analyses of tetra-and penta-silane. J. Chem. Soc., Faraday Trans. 2 1975, 71, 913-920.

(53) Blustin, P. H. A theoretical study of multiple bonding in carbon and silicon. Journal of Organometallic Chemistry 1976, 105, 161 - 168.

(54) Collins, J. B.; von R. Schleyer, P.; Binkley, J. S.; Pople, J. A. Self-consistent molecular orbital methods. XVII. Geometries and binding energies of second-row molecules. A comparison of three basis sets. The Journal of Chemical Physics 1976, 64, 5142-5151. 
(55) Nicolas, G.; Barthelat, J. C.; Durand, P. Valence electronic structure and internal rotation barrier of the molecules XH3YH3 (X, Y = carbon, silicon, germanium) by a pseudopotential method. Journal of the American Chemical Society 1976, 98, 1346-1350.

(56) Berkovitch-yellin, Z.; Ellis, D.; Ratner, M. A. Intramolecular electron localization and local-density calculations on silicon-containing molecules: Tetramethylsilane and hexamethyldisilane. Chemical Physics 1981, 62, $21-35$.

(57) Halevi, E.; Winkelhofer, G.; Meisl, M.; Janoschek, R. Electronic transitions of polysilanes and their photochemistry. Journal of Organometallic Chemistry 1985, 294, 151 - 161.

(58) Luke, B. T.; Pople, J. A.; Krogh-Jespersen, M. B.; Apeloig, Y.; Chandrasekhar, J.; Schleyer, P. v. R. A theoretical survey of singly bonded silicon compounds. Comparison of the structures and bond energies of silyl and methyl derivatives. Journal of the American Chemical Society 1986 , 108, 260-269.

(59) Ortiz, J. V.; Mintmire, J. W. Ground states and ionization energies of Si2H6, Si3H8, Si4H10, and Si5H12. Journal of the American Chemical Society 1988, 110, 4522-4527.

(60) Kawai, E.; Kasatani, K.; Kawasaki, M.; Sato, H.; Hirao, K. Vacuum UV Photoabsorption Spectra of Silane and Disilane: Molecular Orbital Calculation of Electronic States. Japanese Journal of Applied Physics 1989, 28, 247.

(61) Rohlfing, M.; Louie, S. G. Excitonic Effects and the Optical Absorption Spectrum of Hydrogenated Si Clusters. Phys. Rev. Lett. 1998, 80, 3320-3323.

(62) Priya, P. K.; Rai, D. K.; Shukla, A. Photoabsorption in sodium clusters: first principles configuration interaction calculations. Eur. Phys. J. D 2017, 71, 116.

(63) Shinde, R.; Shukla, A. First principles electron-correlated calculations of optical absorption in magnesium clusters. Eur. Phys. J. D 2017, 71, 301.

(64) Ravindra, S.; Alok, S. Large-scale first principles configuration interaction calculations of optical absorption in boron clusters. Nano LIFE 2012, 02, 1240004. 
(65) Ravindra, S.; Alok, S. Large-scale first principles configuration interaction calculations of optical absorption in aluminum clusters. Phys. Chem. Chem. Phys. 2014, 16, 20714-20723.

(66) Aryanpour, K.; Shukla, A.; Mazumdar, S. Electron correlations and two-photon states in polycyclic aromatic hydrocarbon molecules: A peculiar role of geometry. The Journal of Chemical Physics $2014,140,-$.

(67) Shukla, A. Correlated theory of triplet photoinduced absorption in phenylene-vinylene chains. Phys. Rev. B 2002, 65, 125204.

(68) Shukla, A. Theory of nonlinear optical properties of phenyl-substituted polyacetylenes. Phys. Rev. B 2004, 69, 165218 .

(69) Chakraborty, H.; Shukla, A. Pariser-Parr-Pople Model Based Investigation of Ground and LowLying Excited States of Long Acenes. The Journal of Physical Chemistry A 2013, 117, 14220 14229.

(70) Chakraborty, H.; Shukla, A. Theory of triplet optical absorption in oligoacenes: From naphthalene to heptacene. The Journal of Chemical Physics 2014, 141,-.

(71) Sony, P.; Shukla, A. Large-scale correlated calculations of linear optical absorption and low-lying excited states of polyacenes: Pariser-Parr-Pople Hamiltonian. Phys. Rev. B 2007, 75, 155208.

(72) Rai, D. K.; Chakraborty, H.; Shukla, A. Tunable Optoelectronic Properties of Triply Bonded Carbon Molecules with Linear and Graphyne Substructures. The Journal of Physical Chemistry C 2018, 122, 1309-1317.

(73) Basak, T.; Chakraborty, H.; Shukla, A. Theory of linear optical absorption in diamond-shaped graphene quantum dots. Phys. Rev. B 2015, 92, 205404.

(74) Turney, J. M. et al. Psi4: An open-source ab initio electronic structure program. WIREs Comput. Mol. Sci. 2012, 2, 556.

(75) McMurchie, L. E.; Elbert, S. T.; Langhoff, S. R.; Davidson, E. R. MELD package from Indiana University. It has been modified by us to handle bigger systems. 
(76) Frisch, M. J. et al. Gaussian 16 Revision B.01. 2016; Gaussian Inc. Wallingford CT.

(77) Dennington, R.; Keith, T. A.; Millam, J. M. GaussView Version 6. 2016; Semichem Inc. Shawnee Mission KS.

(78) Kokalj, A. XCrySDen-a new program for displaying crystalline structures and electron densities. Journal of Molecular Graphics and Modelling 1999, 17, 176 - 179.

(79) Beagley, B.; Conrad, A.; Freeman, J.; Monaghan, J.; Norton, B.; Holywell, G. Electron diffraction studies of the hydrides Si2H6 and P2H4. Journal of Molecular Structure 1972, 11, 371 - 380.

(80) Shotton, K. C.; Lee, A. G.; Jones, W. J. The pure-rotational Raman spectrum of disilane. Journal of Raman Spectroscopy 1973, 1, 243-253.

(81) Adamczyk, A. J.; Broadbelt, L. J. The Role of Multifunctional Kinetics during Early-Stage Silicon Hydride Pyrolysis: Reactivity of Si2H2 Isomers with SiH4 and Si2H6. The Journal of Physical Chemistry A 2011, 115, 2409-2422, PMID: 21361329.

(82) Koseki, S.; Gordon, M. S. Intrinsic reaction coordinate calculations for very flat potential energy surfaces: application to singlet disilenylidene isomerization. The Journal of Physical Chemistry 1989, 93, 118-125. 


\section{Supporting Information \\ Systematic First Principles Configuration-Interaction Calculations of Linear Optical Absorption Spectra in Silicon Hydrides : $\mathbf{S i}_{2} \mathbf{H}_{2 n}(n=1-3)$}

Pritam Bhattacharyya, ${ }^{*}$ Deepak Kumar Rai, ${ }^{\dagger}$ and Alok Shukla ${ }^{\ddagger}$

Department of Physics, Indian Institute of Technology Bombay, Powai, Mumbai 400076, INDIA

ก

* pritam.bhattacharyya01@gmail.com

$\dagger$ dkriitb@gmail.com

$\ddagger$ shukla@phy.iitb.ac.in 


\section{EXCITED STATE WAVE-FUNCTIONS}

In this section we present important information about the excited states contributing to the peaks in the absorption spectra computed of various conformers of $\mathrm{Si}_{2} \mathrm{H}_{2}, \mathrm{Si}_{2} \mathrm{H}_{4}$, and $\mathrm{Si}_{2} \mathrm{H}_{6}$, using the MRSDCI approach. It consists of plots of frontier molecular orbitals, excitation energies, oscillator strengths, dominant terms in their many-body wave functions, and polarization directions of absorbed photons.

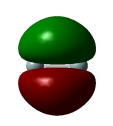

H-3

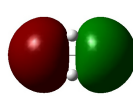

H-2

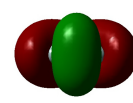

H-1

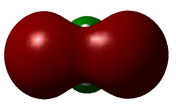

$\mathbf{H}$

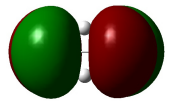

$\mathbf{L}$

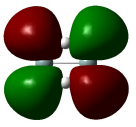

$\mathbf{L}+\mathbf{1}$

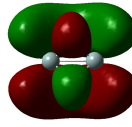

$\mathbf{L}+\mathbf{2}$

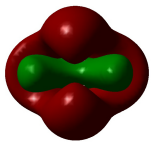

$\mathbf{L}+\mathbf{3}$

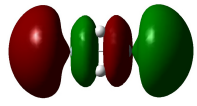

$\mathbf{L}+4$

Figure S1: Various frontier molecular orbitals of dibridged disilyne isomer [main article, Fig. 4(a)] of $\mathrm{Si}_{2} \mathrm{H}_{2}$ molecule, contributing to optical transitions of comparatively high oscillator strength. Symbols H and L denote HOMO and LUMO orbitals, while H-n $(\mathrm{L}+\mathrm{n})$ represents n-th orbital below (above) HOMO (LUMO).

Table S1: Many-particle wave functions of the excited states contributing to the peaks in the optical absorption spectrum of dibridged disilyne $\left(\mathrm{Si}-\mathrm{H}_{2}-\mathrm{Si}\right.$ ) isomer [main article, Fig. 4(a)] of $\mathrm{Si}_{2} \mathrm{H}_{2}$ molecule. 'E' corresponds to excitation energy (in eV) of an excited state, and $f\left(=\frac{2 \times m_{e}}{3 \times \hbar^{2}}(E) \sum_{j=x, y, z}\left|\left\langle e\left|O_{j}\right| R\right\rangle\right|^{2}\right)$ denotes the oscillator strength for a particular electric dipole transition, where $|e\rangle,|R\rangle$ and $O_{j}$ indicate the excited states of the corresponding peak, reference state, and electric dipole operator for different Cartesian components, respectively. In the "|TDM|" column, we present the magnitudes of the transition dipole moments (TDMs) to understand the extent of coupling between the relevant excited state and the ground state. In the "Polarization" column, $\mathrm{x}, \mathrm{y}$ and $\mathrm{z}$ denote the absorption of light polarized along $\mathrm{x}, \mathrm{y}$, and $\mathrm{z}$ directions, respectively. 'HF' corresponds to Hartree-Fock configuration. ' $H$ ' and ' $L$ ' stand for HOMO and LUMO orbitals. In the "Wave function" column, each number inside the parentheses denotes the coefficient of the corresponding configuration in the CI wave function. GS indicates the ground states wave function of the isomer, and not that of an excited state corresponding to a peak. The atomic coordinates corresponding to the optimized ground state geometry of this isomer are presented in Table S9.

\begin{tabular}{|c|c|c|c|c|c|}
\hline Peak & $\mathrm{E}(\mathrm{eV})$ & $f$ & $|\mathrm{TDM}|$ & Polarization & Wave function \\
\hline GS & & & & & $\begin{array}{c}H F\rangle(0.9190) \\
|H \rightarrow L ; H \rightarrow L\rangle(0.0685)\end{array}$ \\
\hline I & 3.360 & 1.37 & 0.7821 & $y$ & $\begin{array}{c}|H \rightarrow L\rangle(0.8945) \\
|H \rightarrow L+12\rangle(0.1101)\end{array}$ \\
\hline II & 4.604 & 0.23 & 0.2738 & $x$ & $\begin{array}{c}|H \rightarrow L+2\rangle(0.8769) \\
|H-1 \rightarrow L ; H \rightarrow L+1\rangle(0.1450)\end{array}$ \\
\hline \multirow[t]{2}{*}{ III } & 5.734 & 13.049 & 1.8476 & $y$ & $\begin{array}{l}|H-1 \rightarrow L\rangle(0.7791) \\
|H \rightarrow L+4\rangle(0.3966)\end{array}$ \\
\hline & 5.768 & 0.352 & 0.3024 & $z$ & $\begin{array}{l}|H \rightarrow L+3 ; H \rightarrow L+3\rangle(0.6692) \\
|H \rightarrow L ; H \rightarrow L\rangle(0.5101)\end{array}$ \\
\hline IV & 6.986 & 13.818 & 1.7225 & $y$ & $\begin{array}{c}|H \rightarrow L+4\rangle(0.7267) \\
|H-3 \rightarrow L+1\rangle(0.4110)\end{array}$ \\
\hline \multirow[t]{2}{*}{ V } & 7.914 & 1.018 & 0.4393 & $z$ & $\begin{aligned} \mid H-1 & \rightarrow L+3 ; H-1 \rightarrow L+3\rangle(0.7953) \\
\mid H & \rightarrow L+5 ; H \rightarrow L+5\rangle(0.2791)\end{aligned}$ \\
\hline & 7.942 & 3.719 & 0.8381 & $x$ & $\begin{array}{l}|H-1 \rightarrow L+2\rangle(0.5350) \\
|H-2 \rightarrow L+1\rangle(0.4774)\end{array}$ \\
\hline
\end{tabular}




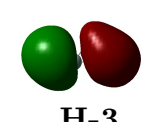

H-3

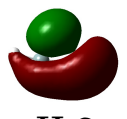

H-2

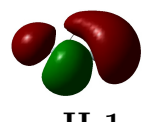

H-1

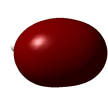

H

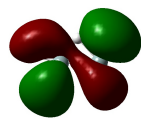

L

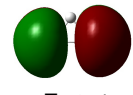

$\mathbf{L}+\mathbf{1}$

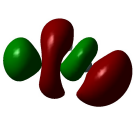

$\mathbf{L}+\mathbf{2}$

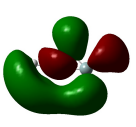

$\mathbf{L}+3$

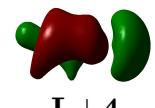

$\mathrm{L}+4$

Figure S2: Various frontier molecular orbitals of mono-bridged isomer [main article, Fig. 4(b)] of $\mathrm{Si}_{2} \mathrm{H}_{2}$ molecule, contributing to optical transitions of comparatively high oscillator strength. Symbols H and L denote the HOMO and the LUMO orbitals, while H-n $(\mathrm{L}+\mathrm{n})$ represents n-th orbital below (above) HOMO (LUMO).

Table S2: Many-particle wave functions of the excited states contributing to the peaks in the optical absorption spectrum of mono-bridged ( $\mathrm{Si}-\mathrm{H}-\mathrm{SiH}$ ) isomer [main article, Fig. 4(b)] of $\mathrm{Si}_{2} \mathrm{H}_{2}$ molecule. 'E' corresponds to excitation energy (in eV) of an excited state, whereas $f$, "TDM", and "Polarization" are defined in the caption of Table S1. 'HF' corresponds to Hartree-Fock configuration. ' $H$ ' and ' $L$ ' stand for HOMO and LUMO orbitals. In the "Wave function" column, each number inside the parentheses denotes the coefficient of the corresponding configuration in the CI wave function. GS indicates the ground states wave function of the isomer, and not that of an excited state corresponding to a peak. The atomic coordinates corresponding to the optimized ground state geometry of this isomer are presented in Table S10.

\begin{tabular}{|c|c|c|c|c|c|}
\hline Peak & $\mathrm{E}(\mathrm{eV})$ & $f$ & $|\mathrm{TDM}|$ & Polarization & Wave function \\
\hline GS & & & & & $\begin{array}{c}|H F\rangle(0.9044) \\
|H \rightarrow L+1 ; H \rightarrow L+1\rangle(0.1311)\end{array}$ \\
\hline I & 2.81 & 0.082 & 0.2094 & $\mathrm{y}$ & $\begin{array}{c}|H \rightarrow L\rangle(0.8984) \\
|H \rightarrow L+2\rangle(0.0859)\end{array}$ \\
\hline \multirow[t]{2}{*}{ II } & 4.01 & 0.755 & $0.25 / 0.47$ & $\mathrm{x} / \mathrm{z}$ & $\begin{array}{l}|H \rightarrow L+1\rangle(0.7516) \\
|H-1 \rightarrow L\rangle(0.4596)\end{array}$ \\
\hline & 4.14 & 0.655 & 0.4861 & $\mathrm{y}$ & $\begin{array}{l}|H \rightarrow L+2\rangle(0.8654) \\
|H \rightarrow L+4\rangle(0.1240)\end{array}$ \\
\hline III & 5.16 & 3.462 & $0.97 / 0.25$ & $\mathrm{x} / \mathrm{z}$ & $\begin{array}{l}|H-1 \rightarrow L\rangle(0.6557) \\
|H \rightarrow L+1\rangle(0.3348)\end{array}$ \\
\hline IV & 5.75 & 0.939 & $0.39 / 0.31$ & $\mathrm{x} / \mathrm{z}$ & $\begin{array}{l}|H-1 \rightarrow L+2\rangle(0.6559) \\
\quad|H-2 \rightarrow L\rangle(0.5002)\end{array}$ \\
\hline V & 6.80 & 4.753 & $0.95 / 0.38$ & $\mathrm{x} / \mathrm{z}$ & $\begin{array}{c}|H-2 \rightarrow L\rangle(0.4030) \\
|H-1 \rightarrow L+2\rangle(0.3875)\end{array}$ \\
\hline VI & 7.22 & 6.728 & 1.1818 & $\mathrm{y}$ & $\begin{aligned} & |H \rightarrow L+4\rangle(0.6325) \\
\mid H \rightarrow & L+1 ; H \rightarrow L+2\rangle(0.3402)\end{aligned}$ \\
\hline VII & 7.66 & 5.660 & $0.77 / 0.72$ & $\mathrm{x} / \mathrm{z}$ & $\begin{array}{l}|H \rightarrow L ; H-1 \rightarrow L+1\rangle(0.4170) \\
\quad|H \rightarrow L ; H \rightarrow L+2\rangle(0.3670)\end{array}$ \\
\hline VIII & 7.91 & 12.025 & $1.09 / 1.03$ & $\mathrm{x} / \mathrm{z}$ & $\begin{array}{l}|H-1 \rightarrow L+4\rangle(0.3523) \\
|H-2 \rightarrow L+2\rangle(0.3201)\end{array}$ \\
\hline IX & 8.86 & 11.742 & $0.79 / 1.17$ & $\mathrm{x} / \mathrm{z}$ & $\begin{array}{c}|H-1 \rightarrow L+4\rangle(0.4106) \\
|H-3 \rightarrow L\rangle(0.3441)\end{array}$ \\
\hline $\mathrm{X}$ & 9.16 & 5.864 & $0.34 / 0.92$ & $\mathrm{x} / \mathrm{z}$ & $\begin{array}{c}|H \rightarrow L+1 ; H-1 \rightarrow L+2\rangle(0.4375) \\
|H \rightarrow L+2 ; H \rightarrow L+2\rangle(0.2899)\end{array}$ \\
\hline
\end{tabular}




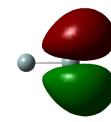

H-2

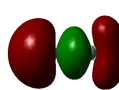

H-1

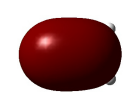

$\mathbf{H}$

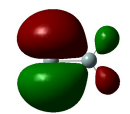

L

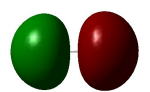

$\mathbf{L}+\mathbf{1}$

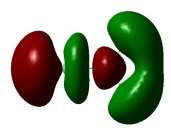

$\mathbf{L}+\mathbf{2}$

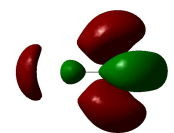

$\mathbf{L}+\mathbf{3}$

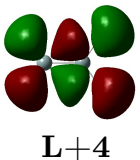

$\mathbf{L}+4$

Figure S3: Various frontier molecular orbitals of disilavinylidene isomer [main article, Fig. 4(c)] of Si $\mathrm{H}_{2} \mathrm{molecule}$ contributing to optical transitions of comparatively high oscillator strength. Symbols H and L denote the HOMO and the LUMO orbitals, while $\mathrm{H}-\mathrm{n}(\mathrm{L}+\mathrm{n})$ represents $\mathrm{n}$-th orbital below (above) HOMO (LUMO).

Table S3: Many-particle wave functions of the excited states contributing to the peaks in the optical absorption spectrum of disilavinylidene ( $\mathrm{Si}-\mathrm{SiH}_{2}$ ) isomer [main article, Fig. 4(c)] of $\mathrm{Si}_{2} \mathrm{H}_{2}$ molecule. 'E' corresponds to excitation energy (in eV) of an excited state, whereas $f$, "TDM", and "Polarization" are defined in the caption of Table S1. 'HF' corresponds to Hartree-Fock configuration. ' $H$ ' and ' $L$ ' stand for HOMO and LUMO orbitals. In the "Wave function" column, each number inside the parentheses denotes the coefficient of the corresponding configuration in the CI wave function. GS indicates the ground states wave function of the isomer, and not that of an excited state corresponding to a peak. The atomic coordinates corresponding to the optimized ground state geometry of this isomer are presented in Table S11.

\begin{tabular}{|c|c|c|c|c|c|}
\hline Peak & $\mathrm{E}(\mathrm{eV})$ & $f$ & $|\mathrm{TDM}|$ & Polarization & Wave function \\
\hline GS & & & & & $\begin{array}{c}|H F\rangle(0.9027) \\
|H \rightarrow L+1 ; H \rightarrow L+1\rangle(0.1626)\end{array}$ \\
\hline I & 2.48 & 0.120 & 0.2699 & $x$ & $\begin{array}{c}|H-1 \rightarrow L\rangle(0.8898) \\
|H-1 \rightarrow L ; H \rightarrow L+1 ; H \rightarrow L+1\rangle(0.1306)\end{array}$ \\
\hline II & 3.13 & 0.213 & 0.3196 & $y$ & $\begin{array}{c}|H \rightarrow L ; H \rightarrow L\rangle(0.8578) \\
|H \rightarrow L+1\rangle(0.1760)\end{array}$ \\
\hline III & 4.29 & 3.820 & 1.1558 & $y$ & $\begin{array}{c}|H \rightarrow L+1\rangle(0.8258) \\
|H-1 \rightarrow L+2\rangle(0.2004)\end{array}$ \\
\hline IV & 5.79 & 1.064 & 0.5250 & $y$ & $\begin{aligned} &|H-2 \rightarrow L\rangle(0.7332) \\
&|H \rightarrow L ; H-2 \rightarrow L+1\rangle(0.3659)\end{aligned}$ \\
\hline V & 6.43 & 1.783 & 0.6450 & $z$ & $\begin{array}{l}|H \rightarrow L+3\rangle(0.6899) \\
|H \rightarrow L+4\rangle(0.4886)\end{array}$ \\
\hline VI & 7.10 & 8.653 & 1.3521 & $z$ & $\begin{array}{l}|H \rightarrow L+4\rangle(0.5807) \\
|H \rightarrow L+3\rangle(0.5414)\end{array}$ \\
\hline VII & 7.57 & 19.917 & 1.9866 & $y$ & $\begin{aligned} \mid H-1 & \rightarrow L+2\rangle(0.6371) \\
\mid H-1 & \rightarrow L+4\rangle(0.4699)\end{aligned}$ \\
\hline VIII & 7.89 & 6.977 & 1.1518 & $y$ & $\begin{aligned} \mid H-1 & \rightarrow L+3\rangle(0.8021) \\
\mid H-1 & \rightarrow L+2\rangle(0.2299)\end{aligned}$ \\
\hline IX & 8.51 & 1.821 & 0.5665 & $y$ & $\begin{array}{c}|H \rightarrow L ; H-2 \rightarrow L+1\rangle(0.7731) \\
|H-1 \rightarrow L+4\rangle(0.2011)\end{array}$ \\
\hline
\end{tabular}




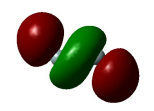

H-2

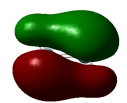

H-1

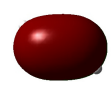

$\mathbf{H}$

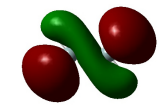

$\mathbf{L}$

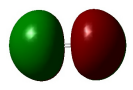

$\mathbf{L}+\mathbf{1}$

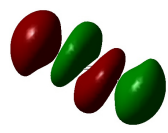

$\mathbf{L}+\mathbf{2}$

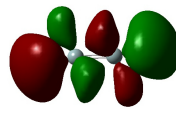

$\mathbf{L}+\mathbf{3}$

Figure S4: Various frontier molecular orbitals of trans-bent isomer [main article, Fig. 4(d)] of $\mathrm{Si}_{2} \mathrm{H}_{2} \mathrm{molecule}_{\text {, contributing }}$ to optical transitions of comparatively high oscillator strength. Symbols H and L denote the HOMO and the LUMO orbitals, while $\mathrm{H}-\mathrm{n}(\mathrm{L}+\mathrm{n})$ represents $\mathrm{n}$-th orbital below (above) HOMO (LUMO).

Table S4: Many-particle wave functions of the excited states contributing to the peaks in the optical absorption spectrum of trans-bent (HSi-SiH) isomer [main article, Fig. 4(d)] of $\mathrm{Si}_{2} \mathrm{H}_{2}$ molecule. 'E' corresponds to excitation energy (in $\mathrm{eV}$ ) of an excited state, whereas $f$, and "TDM" are defined in the caption of Table S1. In the Palarization column, $\perp$ and $\|$ sign indicate the absorption due to the light polarized perpendicularly, and along the plane of the isomer, respectively. 'HF' corresponds to Hartree-Fock configuration. ' $H$ ' and ' $L$ ' stand for HOMO and LUMO orbitals. In the "Wave function" column, each number inside the parentheses denotes the coefficient of the corresponding configuration in the CI wave function. GS indicates the ground states wave function of the isomer, and not that of an excited state corresponding to a peak. The atomic coordinates corresponding to the optimized ground state geometry of this isomer are presented in Table S12.

\begin{tabular}{|c|c|c|c|c|c|}
\hline Peak & $\mathrm{E}(\mathrm{eV})$ & $f$ & $|\mathrm{TDM}|$ & Polarization & Wave function \\
\hline GS & & & & & $\begin{array}{c}|H F\rangle(0.8889) \\
|H-1 \rightarrow L+1\rangle(0.1945)\end{array}$ \\
\hline I & 1.161 & 0.210 & 0.5214 & $\perp$ & $\begin{array}{c}|H \rightarrow L\rangle(0.9045) \\
|H \rightarrow L+11\rangle(0.0871)\end{array}$ \\
\hline II & 2.965 & 10.079 & $2.24 / 0.29$ & $\|$ & $\begin{array}{l}|H-1 \rightarrow L\rangle(0.6892) \\
|H \rightarrow L+1\rangle(0.5865)\end{array}$ \\
\hline III & 4.561 & 0.873 & 0.5357 & $\perp$ & $\begin{array}{c}|H-1 \rightarrow L+1\rangle(0.8919) \\
|H-1 \rightarrow L+14\rangle(0.1047)\end{array}$ \\
\hline IV & 5.047 & 1.363 & $0.54 / 0.34$ & $\|$ & $\begin{array}{l}|H \rightarrow L+1\rangle(0.6117) \\
|H-1 \rightarrow L\rangle(0.5007)\end{array}$ \\
\hline V & 6.004 & 0.411 & $0.19 / 0.26$ & $\|$ & $\begin{array}{l}|H \rightarrow L ; H \rightarrow L+2\rangle(0.8233) \\
|H \rightarrow L ; H \rightarrow L+4\rangle(0.1640)\end{array}$ \\
\hline VI & 6.276 & 3.976 & 0.9748 & $\perp$ & $\begin{array}{c}|H-1 \rightarrow L ; H \rightarrow L+2\rangle(0.5882) \\
|H \rightarrow L+3\rangle(0.4964)\end{array}$ \\
\hline VII & 6.585 & 3.905 & 0.9432 & $\perp$ & $\begin{array}{c}|H \rightarrow L+3\rangle(0.6696) \\
|H-1 \rightarrow L ; H \rightarrow L+2\rangle(0.5031)\end{array}$ \\
\hline VIII & 7.087 & 0.474 & 0.3168 & $\perp$ & $\begin{array}{c}|H-2 \rightarrow L ; H \rightarrow L\rangle(0.6953) \\
|H \rightarrow L+2 ; H \rightarrow L+1\rangle(0.3095)\end{array}$ \\
\hline \multirow[t]{2}{*}{ IX } & 7.323 & 1.140 & 0.4833 & $\perp$ & $\begin{array}{c}|H-2 \rightarrow L ; H \rightarrow L\rangle(0.4417) \\
|H \rightarrow L+2 ; H \rightarrow L+1\rangle(0.4411)\end{array}$ \\
\hline & 7.419 & 0.466 & $0.20 / 0.23$ & $\|$ & $\begin{array}{l}|H-2 \rightarrow L ; H-1 \rightarrow L\rangle(0.6161) \\
|H-2 \rightarrow L+1 ; H \rightarrow L\rangle(0.4922)\end{array}$ \\
\hline
\end{tabular}




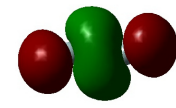

H-1

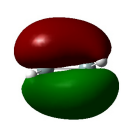

$\mathbf{H}$

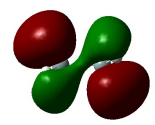

$\mathbf{L}$

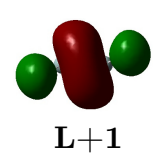

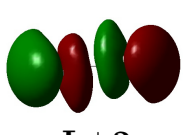

$\mathbf{L}+\mathbf{2}$

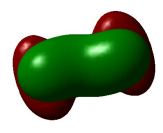

$\mathbf{L}+\mathbf{3}$

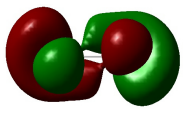

$\mathbf{L}+4$

Figure S5: Various frontier molecular orbitals of disilene isomer [main article, Fig. 9(a)] of $\mathrm{Si}_{2} \mathrm{H}_{4}$ molecule, contributing to optical transitions of comparatively high oscillator strength. Symbols H and L denote the HOMO and the LUMO orbitals, while H-n $(\mathrm{L}+\mathrm{n})$ represents n-th orbital below (above) HOMO (LUMO).

Table S5: Many-particle wave functions of the excited states contributing to the peaks in the optical absorption spectrum of disilene $\left(\mathrm{H}_{2} \mathrm{Si}_{-} \mathrm{SiH}_{2}\right)$ isomer [main article, Fig. 9(a)] of $\mathrm{Si}_{2} \mathrm{H}_{4}$ molecule. 'E' corresponds to excitation energy (in eV) of an excited state, whereas $f$, "TDM", and "Polarization" are defined in the caption of Table S1. 'HF' corresponds to Hartree-Fock configuration. ' $H$ ' and ' $L$ ' stand for HOMO and LUMO orbitals. In the "Wave function" column, each number inside the parentheses denotes the coefficient of the corresponding configuration in the CI wave function. GS indicates the ground states wave function of the isomer, and not that of an excited state corresponding to a peak. The atomic coordinates corresponding to the optimized ground state geometry of this isomer are presented in Table S13.

\begin{tabular}{|c|c|c|c|c|c|}
\hline Peak & $\mathrm{E}(\mathrm{eV})$ & $f$ & $|\mathrm{TDM}|$ & Polarization & Wave function \\
\hline GS & & & & & $\begin{array}{c}|H F\rangle(0.9179) \\
|H \rightarrow L ; H \rightarrow L\rangle(0.1926)\end{array}$ \\
\hline I & 4.132 & 7.816 & $0.32 / 1.65$ & $\mathrm{x} / \mathrm{y}$ & $\begin{aligned} \mid H & \rightarrow L\rangle(0.8879) \\
\mid H-1 & \rightarrow L+2\rangle(0.1258)\end{aligned}$ \\
\hline II & 6.05 & 0.635 & $0.34 / 0.21$ & $\mathrm{x} / \mathrm{y}$ & $\begin{array}{l}|H \rightarrow L+3\rangle(0.7787) \\
|H \rightarrow L+4\rangle(0.4370)\end{array}$ \\
\hline \multirow[t]{2}{*}{ III } & 7.091 & 0.511 & 0.3287 & $\mathrm{z}$ & $\begin{array}{c}|H \rightarrow L ; H \rightarrow L+1\rangle(0.6929) \\
|H \rightarrow L+6\rangle(0.3992)\end{array}$ \\
\hline & 7.309 & 14.375 & $1.72 / 0.04$ & $\mathrm{x} / \mathrm{y}$ & $\begin{array}{l}|H \rightarrow L+4\rangle(0.7321) \\
|H \rightarrow L+3\rangle(0.4620)\end{array}$ \\
\hline
\end{tabular}




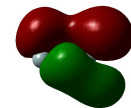

H-3

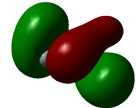

H-2

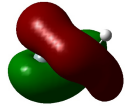

H-1

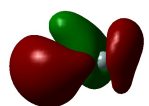

H

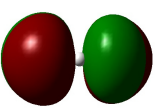

L

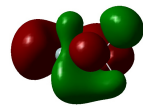

$\mathbf{L}+\mathbf{1}$

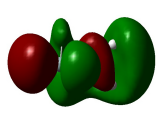

$\mathbf{L}+\mathbf{2}$

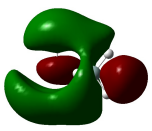

$\mathbf{L}+3$

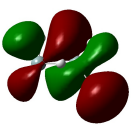

$\mathrm{L}+4$

Figure S6: Various frontier molecular orbitals of mono-bridged isomer [main article, Fig. 9(b)] of $\mathrm{Si}_{2} \mathrm{H}_{4}$ molecule, contributing to optical transitions of comparatively high oscillator strength. Symbols H and L denote the HOMO and the LUMO orbitals, while $\mathrm{H}-\mathrm{n}(\mathrm{L}+\mathrm{n})$ represents n-th orbital below (above) HOMO (LUMO).

Table S6: Many-particle wave functions of the excited states contributing to the peaks in the optical absorption spectrum of mono-bridged $\left(\mathrm{H}_{2} \mathrm{Si}-\mathrm{H}-\mathrm{SiH}\right)$ isomer [main article, Fig. 9(b)] of $\mathrm{Si}_{2} \mathrm{H}_{4}$ molecule. 'E' corresponds to excitation energy (in eV) of an excited state, whereas $f$, "TDM", and "Polarization" are defined in the caption of Table S1. 'HF' corresponds to Hartree-Fock configuration. ' $H$ ' and ' $L$ ' stand for HOMO and LUMO orbitals. In the "Wave function" column, each number inside the parentheses denotes the coefficient of the corresponding configuration in the CI wave function. GS indicates the ground states wave function of the isomer, and not that of an excited state corresponding to a peak. The atomic coordinates corresponding to the optimized ground state geometry of this isomer are presented in Table S14.

\begin{tabular}{|c|c|c|c|c|c|}
\hline Peak & $\mathrm{E}(\mathrm{eV})$ & $f$ & $|\mathrm{TDM}|$ & Polarization & Wave function \\
\hline GS & & & & & $\begin{array}{c}|H F\rangle(0.9248) \\
|H \rightarrow L ; H \rightarrow L\rangle(0.0647)\end{array}$ \\
\hline I & 3.831 & 0.815 & $0.34 / 0.45$ & $\mathrm{x} / \mathrm{z}$ & $\begin{array}{c}|H \rightarrow L\rangle(0.8789) \\
|H \rightarrow L+1\rangle(0.1414)\end{array}$ \\
\hline II & 4.845 & 0.309 & $0.24 / 0.20$ & $\mathrm{x} / \mathrm{z}$ & $\begin{array}{l}|H-1 \rightarrow L\rangle(0.7965) \\
|H \rightarrow L+1\rangle(0.3411)\end{array}$ \\
\hline III & 5.439 & 4.036 & 1.0500 & $\mathrm{x}$ & $\begin{array}{l}|H \rightarrow L+1\rangle(0.7770) \\
|H-1 \rightarrow L\rangle(0.3132)\end{array}$ \\
\hline IV & 6.576 & 0.696 & $0.24 / 0.30$ & $\mathrm{y} / \mathrm{z}$ & $\begin{array}{c}|H-1 \rightarrow L+1\rangle(0.6786) \\
|H \rightarrow L+2\rangle(0.4134)\end{array}$ \\
\hline \multirow[t]{2}{*}{ V } & 6.862 & 1.260 & $0.22 / 0.47$ & $\mathrm{y} / \mathrm{z}$ & $\begin{array}{c}|H \rightarrow L+2\rangle(0.5174) \\
|H-1 \rightarrow L+1\rangle(0.4870)\end{array}$ \\
\hline & 6.976 & 2.346 & 0.7070 & $\mathrm{x}$ & $\begin{array}{l}|H \rightarrow L+3\rangle(0.5177) \\
|H-2 \rightarrow L\rangle(0.5144)\end{array}$ \\
\hline VI & 7.249 & 3.596 & $0.84 / 0.20$ & $\mathrm{x} / \mathrm{z}$ & $\begin{array}{l}|H-2 \rightarrow L\rangle(0.6016) \\
|H \rightarrow L+4\rangle(0.3915)\end{array}$ \\
\hline VII & 7.430 & 2.481 & $0.47 / 0.45 / 0.27$ & $\mathrm{x} / \mathrm{y} / \mathrm{z}$ & $\begin{array}{c}|H \rightarrow L ; H \rightarrow L\rangle(0.5338) \\
|H-3 \rightarrow L\rangle(0.3569)\end{array}$ \\
\hline VIII & 7.801 & 6.823 & $1.02 / 0.50 / 0.18$ & $\mathrm{x} / \mathrm{y} / \mathrm{z}$ & $\begin{array}{l}|H \rightarrow L+4\rangle(0.5845) \\
|H-3 \rightarrow L\rangle(0.4178)\end{array}$ \\
\hline
\end{tabular}




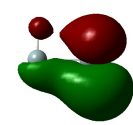

H-2

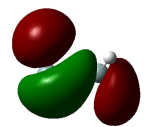

H-1

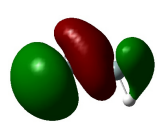

$\mathbf{H}$

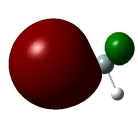

$\mathbf{L}$

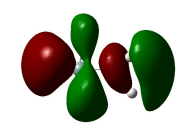

$\mathbf{L}+\mathbf{1}$

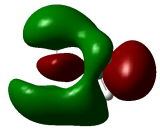

$\mathbf{L}+\mathbf{2}$

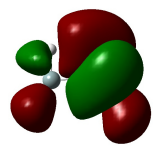

$\mathbf{L}+\mathbf{3}$

Figure S7: Various frontier molecular orbitals of silylsilylene isomer [main article, Fig. 9(c)] of $\mathrm{Si}_{2} \mathrm{H}_{4}$ molecule, contributing to optical transitions of comparatively high oscillator strength. Symbols H and L denote the HOMO and the LUMO orbitals, while $\mathrm{H}-\mathrm{n}(\mathrm{L}+\mathrm{n})$ represents n-th orbital below (above) HOMO (LUMO).

Table S7: Many-particle wave functions of the excited states contributing to the peaks in the optical absorption spectrum of silylsilylene $\left(\mathrm{H}_{3} \mathrm{Si}-\mathrm{SiH}\right)$ isomer [main article, Fig. 9(c)] of $\mathrm{Si}_{2} \mathrm{H}_{4}$ molecule. 'E' corresponds to excitation energy (in eV) of an excited state, whereas $f$, "TDM", and "Polarization" are defined in the caption of Table S1. 'HF" corresponds to Hartree-Fock configuration. ' $H$ ' and ' $L$ ' stand for HOMO and LUMO orbitals. In the "Wave function" column, each number inside the parentheses denotes the coefficient of the corresponding configuration in the CI wave function. GS indicates the ground states wave function of the isomer, and not that of an excited state corresponding to a peak. The atomic coordinates corresponding to the optimized ground state geometry of this isomer are presented in Table S15.

\begin{tabular}{|c|c|c|c|c|c|}
\hline Peak & $\mathrm{E}(\mathrm{eV})$ & $f$ & $|\mathrm{TDM}|$ & Polarization & Wave function \\
\hline GS & & & & & $\begin{aligned} & |H F\rangle(0.9250) \\
\mid H \rightarrow & L ; H \rightarrow L\rangle(0.0978)\end{aligned}$ \\
\hline I & 1.935 & 0.131 & 0.3187 & $\mathrm{z}$ & $\begin{array}{c}|H \rightarrow L\rangle(0.9163) \\
|H \rightarrow L+15\rangle(0.0960)\end{array}$ \\
\hline II & 3.795 & 0.136 & 0.2316 & $\mathrm{z}$ & $\begin{array}{c}|H-1 \rightarrow L\rangle(0.8985) \\
|H-1 \rightarrow L+15\rangle(0.1136)\end{array}$ \\
\hline \multirow[t]{2}{*}{ III } & 6.105 & 0.505 & 0.3522 & $\mathrm{x}$ & $\begin{aligned} \mid H-1 & \rightarrow L ; H \rightarrow L\rangle(0.8374) \\
\mid H & \rightarrow L+1\rangle(0.2040)\end{aligned}$ \\
\hline & 6.257 & 0.216 & 0.2267 & $\mathrm{z}$ & $\begin{array}{c}|H-2 \rightarrow L\rangle(0.8484) \\
|H-2 \rightarrow L ; H \rightarrow L+1\rangle(0.1526)\end{array}$ \\
\hline IV & 6.639 & 1.186 & $0.20 / 0.48$ & $\mathrm{x} / \mathrm{y}$ & $\begin{array}{l}|H \rightarrow L+2\rangle(0.6048) \\
|H \rightarrow L+1\rangle(0.5465)\end{array}$ \\
\hline V & 6.971 & 15.375 & 1.8178 & $\mathrm{x}$ & $\begin{array}{l}|H \rightarrow L+1\rangle(0.5637) \\
|H \rightarrow L+2\rangle(0.5528)\end{array}$ \\
\hline VI & 7.354 & 4.986 & $0.85 / 0.54$ & $\mathrm{x} / \mathrm{y}$ & $\begin{array}{c}|H \rightarrow L+3\rangle(0.7725) \\
|H-1 \rightarrow L+1\rangle(0.2561)\end{array}$ \\
\hline
\end{tabular}




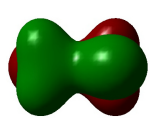

$(\mathrm{H}-2)_{2}$

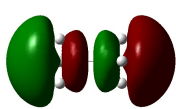

L

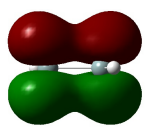

(H-2) 1

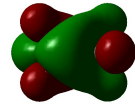

$\mathbf{L}+\mathbf{1}$

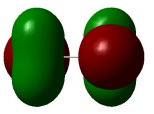

(H-1) 2

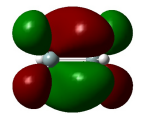

$(\mathrm{L}+\mathbf{2})_{1}$

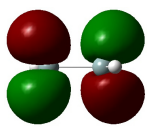

(H-1) 1

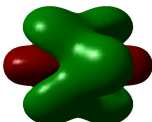

$(\mathrm{L}+2)_{2}$

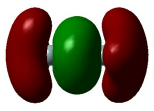

H

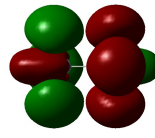

$\mathrm{L}+4$

Figure S8: Various frontier molecular orbitals of disilane isomer [main article, Fig. 2] of $\mathrm{Si}_{2} \mathrm{H}_{6}$ molecule, contributing to optical transitions of comparatively high oscillator strength. Symbols H and L denote the HOMO and the LUMO orbitals, while H-n $(\mathrm{L}+\mathrm{n})$ represents $\mathrm{n}$-th orbital below (above) HOMO (LUMO). Subscripts are used to index the degenerate orbitals.

Table S8: Many-particle wave functions of the excited states contributing to the peaks in the optical absorption spectrum of disilane $\left(\mathrm{H}_{3} \mathrm{Si}_{-} \mathrm{SiH}_{3}\right)$ isomer [main article, Fig. 2] of $\mathrm{Si}_{2} \mathrm{H}_{6}$ molecule. 'E' corresponds to excitation energy (in $\mathrm{eV}$ ) of an excited state, whereas $f$, "TDM", and "Polarization" are defined in the caption of Table S1. 'HF' corresponds to Hartree-Fock configuration. ' $H$ ' and ' $L$ ' stand for HOMO and LUMO orbitals. In the "Wave function" column, each number inside the parentheses denotes the coefficient of the corresponding configuration in the CI wave function. GS indicates the ground states wave function of the isomer, and not that of an excited state corresponding to a peak. The atomic coordinates corresponding to the optimized ground state geometry of this isomer are presented in Table S16.

\begin{tabular}{|c|c|c|c|c|c|}
\hline Peak & $\mathrm{E}(\mathrm{eV})$ & $f$ & $|\mathrm{TDM}|$ & Polarization & Wave function \\
\hline GS & & & & & $|H F\rangle(0.9409)$ \\
\hline \multirow[t]{2}{*}{ I } & 7.767 & 2.976 & $0.74 / 0.18$ & $\mathrm{x} / \mathrm{y}$ & $\begin{array}{l}\left|H \rightarrow(L+2)_{2}\right\rangle(0.8791) \\
\left|(H-1)_{1} \rightarrow L\right\rangle(0.1246)\end{array}$ \\
\hline & 7.812 & 5.984 & 1.0719 & $\mathrm{z}$ & $\begin{array}{l}\left|H \rightarrow(L+2)_{1}\right\rangle(0.8809) \\
\left|(H-2)_{1} \rightarrow L\right\rangle(0.1257)\end{array}$ \\
\hline II & 8.445 & 20.638 & $0.31 / 1.89$ & $\mathrm{x} / \mathrm{y}$ & $\begin{aligned} \mid H & \rightarrow L\rangle(0.8486) \\
\mid(H-1)_{1} & \left.\rightarrow(L+2)_{2}\right\rangle(0.1901)\end{aligned}$ \\
\hline III & 9.208 & 6.959 & 1.0647 & $\mathrm{z}$ & $\begin{array}{l}\left|(H-1)_{1} \rightarrow(L+2)_{1}\right\rangle(0.6114) \\
\left|(H-1)_{2} \rightarrow(L+2)_{2}\right\rangle(0.5962)\end{array}$ \\
\hline \multirow[t]{2}{*}{ IV } & 9.404 & 8.843 & $0.31 / 1.15$ & $\mathrm{x} / \mathrm{y}$ & $\begin{array}{c}\left|(H-1)_{1} \rightarrow L\right\rangle(0.5695) \\
\left|(H-1)_{1} \rightarrow(L+2)_{2}\right\rangle(0.4532)\end{array}$ \\
\hline & 9.471 & 0.800 & 0.3559 & $\mathrm{z}$ & $\begin{array}{c}\left|(H-2)_{1} \rightarrow L\right\rangle(0.5852) \\
\left|(H-1)_{2} \rightarrow(L+2)_{2}\right\rangle(0.4249)\end{array}$ \\
\hline \multirow[t]{2}{*}{$\mathrm{V}$} & 9.816 & 8.668 & $0.62 / 0.97$ & $\mathrm{x} / \mathrm{y}$ & $\begin{array}{c}\left|(H-1)_{1} \rightarrow(L+2)_{2}\right\rangle(0.6397) \\
\quad\left|(H-1)_{1} \rightarrow L\right\rangle(0.3825)\end{array}$ \\
\hline & 9.924 & 1.031 & 0.3947 & $\mathrm{z}$ & $\begin{array}{c}\left|(H-2)_{1} \rightarrow L\right\rangle(0.6167) \\
\left|(H-1)_{2} \rightarrow(L+2)_{2}\right\rangle(0.4176)\end{array}$ \\
\hline VI & 10.161 & 2.259 & $0.53 / 0.22$ & $\mathrm{x} / \mathrm{y}$ & $\begin{array}{c}|H \rightarrow L+4\rangle(0.7547) \\
\left|(H-1)_{2} \rightarrow(L+2)_{1}\right\rangle(0.3177)\end{array}$ \\
\hline
\end{tabular}




\section{INFLUENCE OF BASIS SETS ON THE OPTICAL ABSORPTION SPECTRUM}

We computed the optical absorption spectra of trans-bent (HSi-SiH) molecule using both, cc-pVTZ and aug-ccpVTZ basis sets and MRSDCI methodology to verify whether the computed spectrum has converged with respect to the basis set. The main difference between the spectra computed using two basis sets is the intensity of the main peak. Computed peak positions are in very good agreement.

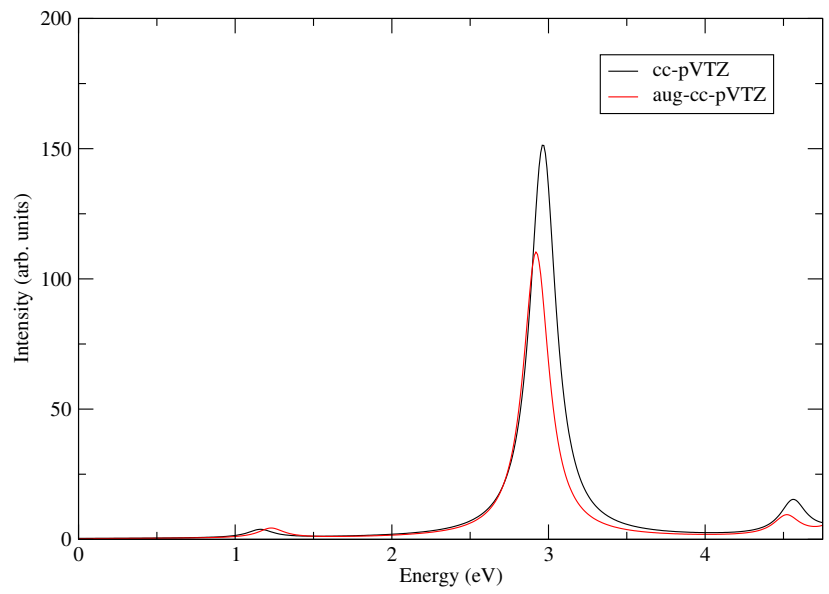

Figure S9: Optical absorption spectrum of trans-bent (HSi-SiH) conformer, calculated using the MRSDCI method, and the cc-pVTZ and aug-cc-pVTZ basis sets. For plotting the spectrum, a uniform line-width of $0.1 \mathrm{eV}$ was used.

\section{OPTIMIZED EXCITED STATE GEOMETRIES}

In this section we present the optimized geometries of various excited states in the optical absorption spectra of various clusters. For details refer to section 2 of the main text.

\section{A. Dibridged disilyne $\left(\mathrm{Si}-\mathrm{H}_{2}-\mathrm{Si}\right)$ isomer}

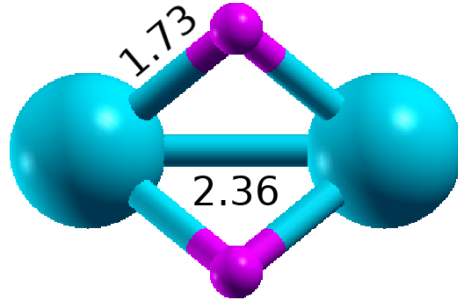

(a) Peak-I

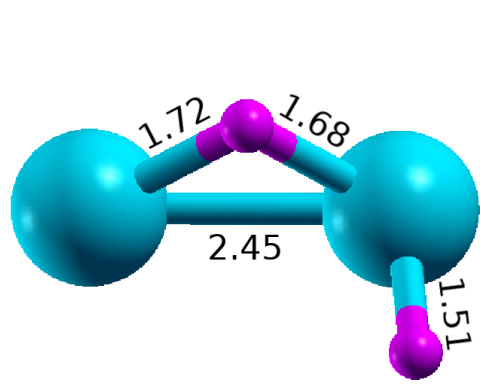

(b) Peak-III

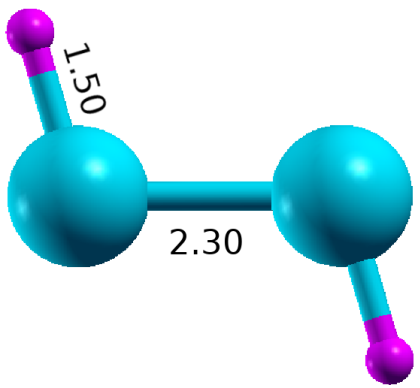

(c) Peak-IV

Figure S10: Optimized geometries of the excited states corresponding to various peaks of the absorption spectrum of dibridged disilyne ( $\mathrm{Si}-\mathrm{H}_{2}-\mathrm{Si}$ ) isomer [main article, Fig. 4(a)]. All the bond lengths are in $\AA$ unit. 


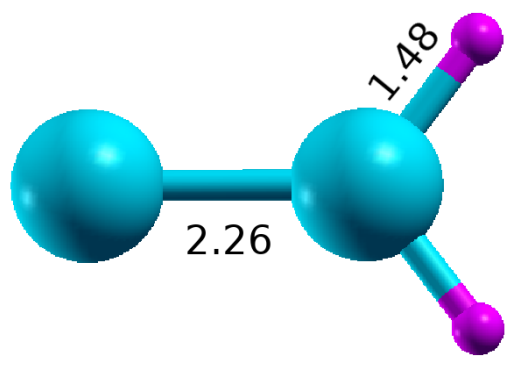

(a) Peak-II

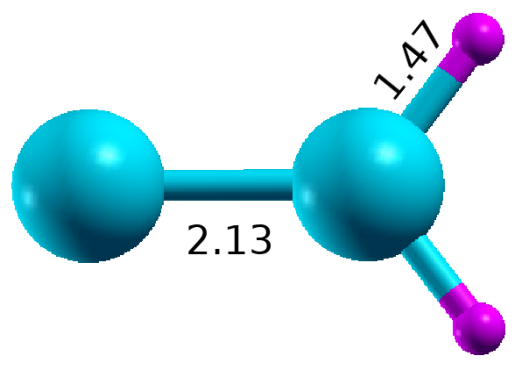

(b) Peak-III

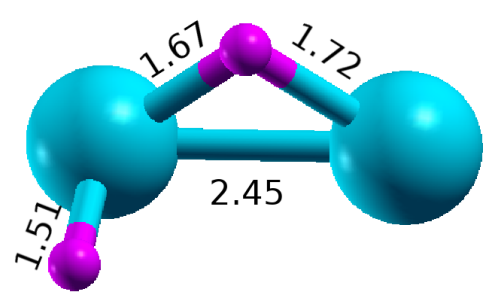

(c) Peak-VIII

Figure S11: Optimized excited state geometries corresponding to various peaks of the absorption spectrum of monobridged (Si-H-SiH) isomer [main article, Fig. 4(b)]. All the bond lengths are in $\AA$ unit.

\section{Disilavinylidene $\left(\mathrm{Si}_{-} \mathrm{SiH}_{2}\right)$ isomer}

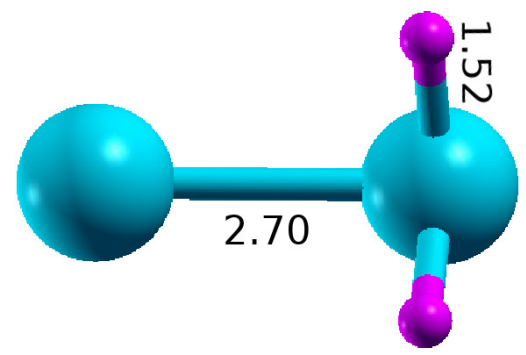

(a) Peak-III

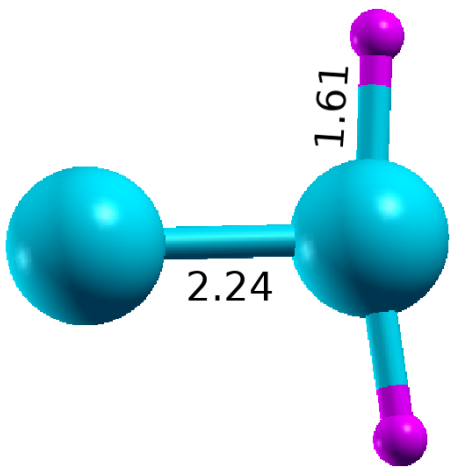

(b) Peak-VII

Figure S12: Optimized excited state geometries corresponding to various peaks of the absorption spectrum of disilavinylidene $\left(\mathrm{Si}_{-} \mathrm{SiH}_{2}\right)$ isomer [main article, Fig. 4(c)]. All the bond lengths are in $\AA$ unit.

\section{Trans-bent (HSi-SiH) isomer}

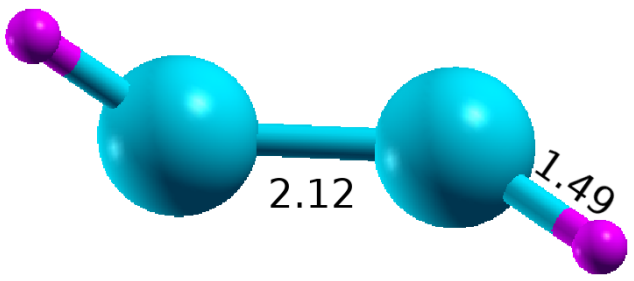

(a) Peak-I

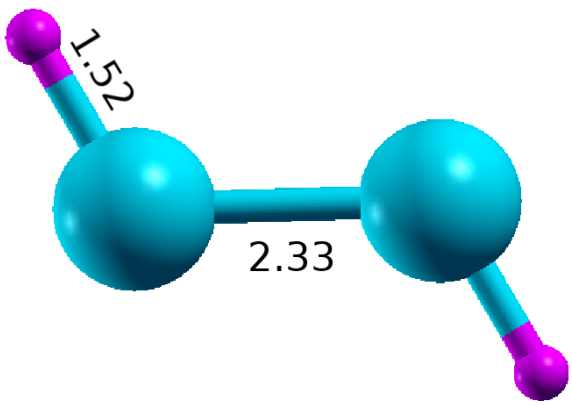

(b) Peak-II

Figure S13: Optimized geometries of excited states corresponding to various peaks of the absorption spectrum of trans-bent (HSi-SiH) isomer [main article, Fig. 4(d)]. All the bond lengths are in $\AA$ unit. 


\section{E. Disilene $\left(\mathrm{H}_{2} \mathrm{Si}-\mathrm{SiH}_{2}\right)$ isomer}

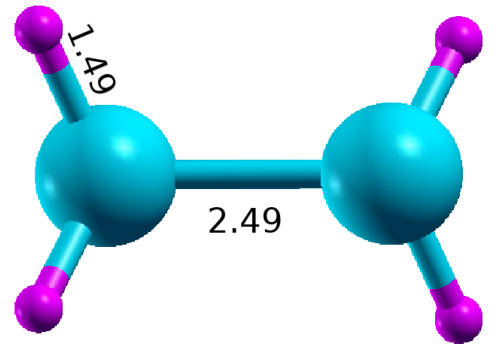

(a) Peak-I

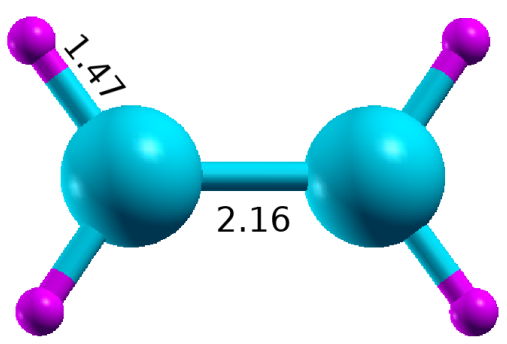

(b) Peak-III

Figure S14: Optimized excited state geometries corresponding to various peaks of the absorption spectrum of disilene $\left(\mathrm{H}_{2} \mathrm{Si}_{-} \mathrm{SiH}_{2}\right)$ isomer [main article, Fig. 9(a)]. All the bond lengths are in $\AA$ unit.

\section{F. Monobridged $\left(\mathrm{H}_{2} \mathrm{Si}-\mathrm{H}-\mathrm{SiH}\right)$ isomer}

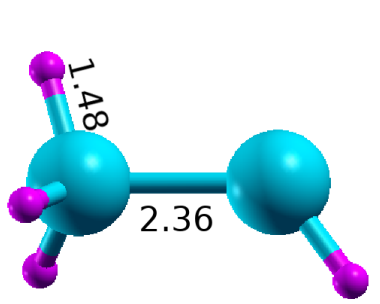

(a) Peak-I

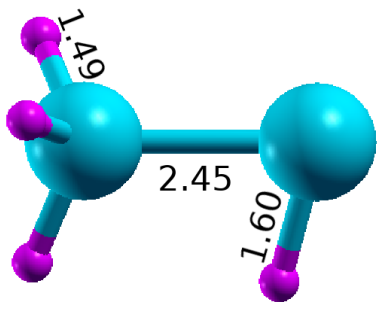

(b) Peak-II

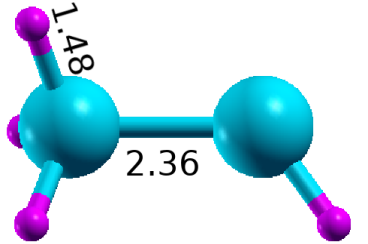

(c) Peak-III

Figure S15: Optimized excited state geometries corresponding to various peaks of the absorption spectrum of monobridged $\left(\mathrm{H}_{2} \mathrm{Si}-\mathrm{H}-\mathrm{SiH}\right)$ isomer [main article, Fig. 9(b)]. All the bond lengths are in $\AA$ unit.

\section{G. Silylsilylene $\left(\mathrm{H}_{3} \mathrm{Si}-\mathrm{SiH}\right)$ isomer}

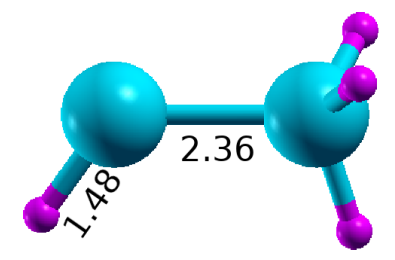

(a) Peak-I

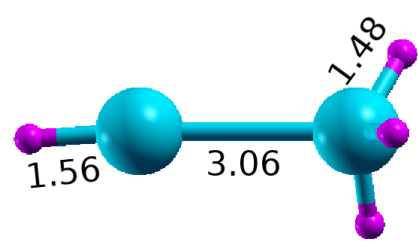

(b) Peak-V

Figure S16: Optimized excited state geometries corresponding to various peaks of the absorption spectrum of silylsilylene $\left(\mathrm{H}_{3} \mathrm{Si}-\mathrm{SiH}\right)$ isomer [main article, Fig. 9(c)]. All the bond lengths are in $\AA$ unit. 
H. Disilane $\left(\mathrm{H}_{3} \mathrm{Si}-\mathrm{SiH}_{3}\right)$ isomer

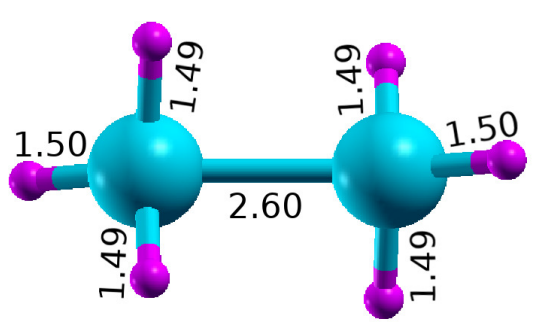

(a) Peak-I

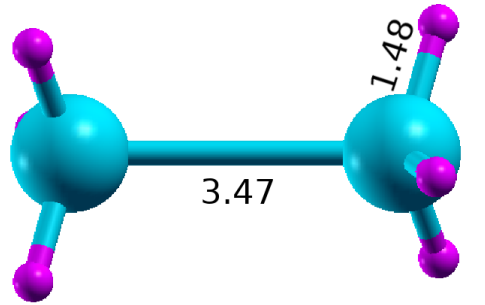

(b) Peak-II

Figure S17: Optimized excited state geometries corresponding to various peaks of the absorption spectrum of disilane $\left(\mathrm{H}_{3} \mathrm{Si}_{-} \mathrm{SiH}_{3}\right)$ isomer [main article, Fig. 2]. All the bond lengths are in $\AA$ unit.

\section{ATOMIC COORDINATES CORRESPONDING TO THE OPTIMIZED GROUND STATE GEOMETRIES OF VARIOUS CLUSTERS}

Table S9: Atomic coordinates (in $\AA$ units) corresponding to the optimized ground state geometry of dibridged disilyne $\left(\mathrm{Si}-\mathrm{H}_{2}-\mathrm{Si}\right)$

\begin{tabular}{cccc}
\hline & $\mathrm{x}$ & $\mathrm{y}$ & $\mathrm{z}$ \\
\hline $\mathrm{Si}$ & 0.000000000000 & 1.100740944342 & -0.027074095829 \\
$\mathrm{Si}$ & 0.000000000000 & -1.100740944342 & -0.027074095829 \\
$\mathrm{H}$ & -0.985364830979 & 0.000000000000 & 0.751568938885 \\
$\mathrm{H}$ & 0.985364830979 & 0.000000000000 & 0.751568938885 \\
\hline
\end{tabular}

Table S10: Atomic coordinates (in $\AA$ units) corresponding to the optimized ground state geometry of monobridged structure $(\mathrm{Si}-\mathrm{H}-\mathrm{SiH})$

\begin{tabular}{cccc}
\hline & $\mathrm{x}$ & $\mathrm{y}$ & $\mathrm{z}$ \\
\hline $\mathrm{Si}$ & -0.836958729655 & 0.017059638406 & -0.709155735004 \\
$\mathrm{Si}$ & 0.806304271215 & -0.014312279108 & 0.606308390319 \\
$\mathrm{H}$ & -0.772104895994 & -0.033803861038 & 1.012668921759 \\
$\mathrm{H}$ & 1.623063648110 & -0.042462025198 & 1.842343123724 \\
\hline
\end{tabular}

Table S11: Atomic coordinates (in $\AA$ units) corresponding to the optimized ground state geometry of disilavinylidene isomer $\left(\mathrm{Si}_{-} \mathrm{SiH}_{2}\right)$

\begin{tabular}{cccc}
\hline & $\mathrm{x}$ & $\mathrm{y}$ & $\mathrm{z}$ \\
\hline $\mathrm{Si}$ & 0.000038658677 & 1.165679188613 & 0.000000000000 \\
$\mathrm{Si}$ & -0.000024520199 & -1.031699608620 & 0.000000000000 \\
$\mathrm{H}$ & 1.227406456834 & -1.859865255861 & 0.000000000000 \\
$\mathrm{H}$ & -1.227798936833 & -1.859368487152 & 0.000000000000 \\
\hline
\end{tabular}


Table S12: Atomic coordinates (in $\AA$ units) corresponding to the optimized ground state geometry of trans-bent isomer $(\mathrm{HSi}-\mathrm{SiH})$

\begin{tabular}{cccc}
\hline & $\mathrm{x}$ & $\mathrm{y}$ & $\mathrm{z}$ \\
\hline $\mathrm{Si}$ & 1.049560638794 & -0.012108929052 & 0.000000000000 \\
$\mathrm{Si}$ & -1.049560638794 & 0.012108929052 & 0.000000000000 \\
$\mathrm{H}$ & 1.901548085640 & -1.229661195450 & 0.000000000000 \\
$\mathrm{H}$ & -1.901548085640 & 1.229661195450 & 0.000000000000 \\
\hline
\end{tabular}

Table S13: Atomic coordinates (in $\AA$ units) corresponding to the optimized ground state geometry of disilene isomer $\left(\mathrm{H}_{2} \mathrm{Si}_{-} \mathrm{SiH}_{2}\right)$

\begin{tabular}{cccc}
\hline \hline & $\mathrm{x}$ & $\mathrm{y}$ & $\mathrm{z}$ \\
\hline $\mathrm{Si}$ & -0.014527084989 & 1.078610931417 & 0.000000000000 \\
$\mathrm{Si}$ & 0.014527084989 & -1.078610931417 & 0.000000000000 \\
$\mathrm{H}$ & -0.388083856897 & 1.807751814186 & 1.227693520012 \\
$\mathrm{H}$ & -0.388083856897 & 1.807751814186 & -1.227693520012 \\
$\mathrm{H}$ & 0.388083856897 & -1.807751814186 & -1.227693520012 \\
$\mathrm{H}$ & 0.388083856897 & -1.807751814186 & 1.227693520012 \\
\hline
\end{tabular}

Table S14: Atomic coordinates (in $\AA$ units) corresponding to the optimized ground state geometry of mono-bridged isomer $\left(\mathrm{H}_{2} \mathrm{Si}-\mathrm{H}-\mathrm{SiH}\right)$

\begin{tabular}{cccc}
\hline & $\mathrm{x}$ & $\mathrm{y}$ & $\mathrm{z}$ \\
\hline $\mathrm{Si}$ & 1.172682525006 & 0.047991888154 & -0.052395704519 \\
$\mathrm{Si}$ & -1.070659956950 & 0.004587875388 & -0.004943355672 \\
$\mathrm{H}$ & 1.079592180677 & -1.466147217376 & -0.026756050259 \\
$\mathrm{H}$ & -0.073757438423 & -0.034913083199 & 1.266626592692 \\
$\mathrm{H}$ & -1.881741837422 & 1.218385602498 & 0.242557859563 \\
$\mathrm{H}$ & -1.956209393611 & -1.176924076404 & 0.109287047982 \\
\hline
\end{tabular}

Table S15: Atomic coordinates (in $\AA$ units) corresponding to the optimized ground state geometry of silylsilylene isomer $\left(\mathrm{H}_{3} \mathrm{Si}-\mathrm{SiH}\right)$

\begin{tabular}{cccc}
\hline & $\mathrm{x}$ & $\mathrm{y}$ & $\mathrm{z}$ \\
\hline $\mathrm{Si}$ & 1.126462143734 & 0.027035432078 & 0.000006113340 \\
$\mathrm{Si}$ & -1.258201977707 & -0.087127476208 & -0.000009612313 \\
$\mathrm{H}$ & -1.308985044826 & 1.431034864085 & 0.000041211523 \\
$\mathrm{H}$ & 1.587300210721 & 0.767155781338 & 1.203027047658 \\
$\mathrm{H}$ & 1.791407763335 & -1.297349115623 & -0.000057042453 \\
$\mathrm{H}$ & 1.587336122859 & 0.767295944767 & -1.202914086260 \\
\hline
\end{tabular}


Table S16: Atomic coordinates (in $\AA$ units) corresponding to the optimized ground state geometry of disilane $\left(\mathrm{H}_{3} \mathrm{Si}\right.$ $\mathrm{SiH}_{3}$ )

\begin{tabular}{lccc}
\hline & $\mathrm{x}$ & $\mathrm{y}$ & $\mathrm{z}$ \\
\hline $\mathrm{Si}$ & 0.000000000915 & -1.169370238817 & 0.000000000000 \\
$\mathrm{Si}$ & -0.000000000915 & 1.169370238817 & 0.000000000000 \\
$\mathrm{H}$ & -1.388422645000 & -1.684057891419 & 0.000000000000 \\
$\mathrm{H}$ & 0.694211326479 & -1.684057901803 & -1.202409278566 \\
$\mathrm{H}$ & 0.694211326479 & -1.684057901803 & 1.202409278566 \\
$\mathrm{H}$ & 1.388422645000 & 1.684057891419 & 0.000000000000 \\
$\mathrm{H}$ & -0.694211326479 & 1.684057901803 & -1.202409278566 \\
$\mathrm{H}$ & -0.694211326479 & 1.684057901803 & 1.202409278566 \\
\hline
\end{tabular}

